\title{
Additions to the lichenized and lichenicolous fungi in Bulgaria
}

\section{Helmut Mayrhofer $^{1 *}$, Adriana Atanassova ${ }^{1}$, Siyka O. Nikolova ${ }^{2} \&$ Cvetomir M. Denchev ${ }^{2}$}

${ }^{1}$ Institute of Biology, Division of Plant Sciences, NAWI Graz, University of Graz, Holteigasse 6, A-8010 Graz, Austria

${ }^{2}$ Institute of Biodiversity and Ecosystem Research, Bulgarian Academy of Sciences, 2 Gagarin St., 1113 Sofia, Bulgaria

Received 28 July 2020 / Accepted 6 August 2020 / Published 7 August 2020

Mayrhofer, H., Atanassova, A., Nikolova, S.O. \& Denchev, C.M. 2020. Additions to the lichenized and lichenicolous fungi in Bulgaria. - Mycobiota 10: 39-62. doi: 10.12664/mycobiota.2020.10.04

\begin{abstract}
Thirty-six taxa of lichenized fungi, Acarospora irregularis, Arthonia mediella, Caloplaca asserigena, C. atroflava, C. subpallida, Catillaria detractula, Diplotomma hedinii, Endohyalina insularis, Lecanora rouxii, L. rupicola subsp. subplanata, Lecidea berengeriana, L. sarcogynoides, Lepra leucosora, Lepraria borealis, L. diffusa, L. elobata, L. nylanderiana, L. vouauxii, Ochrolechia arborea, Pertusaria flavicans, Protoparmeliopsis muralis var. dubyi, Pycnora praestabilis, Rinodina freyi, $R$. luridata var. immersa, $R$. occulta, $R$. roscida, $R$. sicula, $R$. teichophila, $R$. trevisanii, Rinodinella dubyanoides, Scoliciosporum umbrinum var. corticicolum, Solorina bispora var. macrospora, Strigula affinis, Tephromela atra var. torulosa, Umbilicaria freyi, and U. maculata, are reported for the first time from Bulgaria. The finding of Rinodina sicula represents the first record for the Balkan Peninsula.
\end{abstract}

Key words: biodiversity, Bulgaria, lichenicolous fungi, lichenized fungi, Rinodina, taxonomy

\section{Introduction}

The present paper is a contribution to the lichen biota of Bulgaria and the Balkan Peninsula. It includes 36 taxa of lichenized fungi new for Bulgaria. Additionally, some new records for

\footnotetext{
*Corresponding authors: e-mail: helmut.mayrhofer@uni-graz.at
} 
distinct floristic regions are also reported. Eleven taxa previously synonymized by Mayrhofer et al. (2005) are resurrected following recent taxonomic treatments.

\section{Material and methods}

The specimens were collected during three short field trips by H. M. - two with S. O. Nikolova $(2007,2008)$ and one with A. Atanassova (2010) with the main focus on the Physciaceae. Further specimens were collected by A. Atanassova in 2009 and 2011. Most of the specimens are kept in the herbarium GZU. Thin-layer chromatography (TLC) was performed in solvents A, B', and C on selected taxa following Orange et al. (2001). The nomenclature follows Nimis et al. (2018) and for taxa not occurring in the Alps, Nimis (2016). In the case of nomenclatural changes, citations are followed by the name used in the original publication. The authors of taxa follow Nimis et al. (2018).

The genera and taxa within each genus are given in alphabetical order. The numbers within square brackets following the authors refer to the floristic regions of Bulgaria as used by Mayrhofer et al. (2005) from where the taxa are recorded. New records for Bulgaria are indicated by an asterisk $(*)$. Corrections to taxa, published in Catalogue of the lichenized and lichenicolous fungi in Bulgaria (Mayrhofer et al. 2005), are indicated by a hash (\#).

\section{Results}

\section{Lichenized fungi}

Acarospora cervina (Ach.) A. Massal. [5, 18]

Balkan Range, Mt. Ponor, Sofia Province, Dobravitsa village, Topilata neighbourhood, on

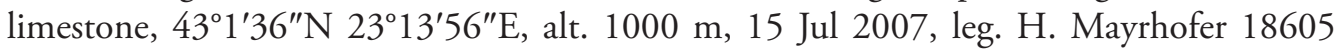
$\&$ S. Nikolova; Thracian Lowland, Plovdiv Province, Besaparski Ridove, on limestone, $42^{\circ} 5^{\prime} 13^{\prime \prime} \mathrm{N} 24^{\circ} 28^{\prime} 47^{\prime \prime} \mathrm{E}$, alt. 220 m, 13 May 2008, leg. H. Mayrhofer 18567 \& S. Nikolova.

\section{*Acarospora irregularis H. Magn. [17]}

The Rhodopes, Kurdzhali Province, dam of Stouden Kladenets Reservoir, on trachyte, $41^{\circ} 36^{\prime} 59^{\prime \prime} \mathrm{N} 25^{\circ} 38^{\prime} 30^{\prime \prime}$, alt. 230 m, 14 May 2008, leg. H. Mayrhofer 18537 \& S. Nikolova, det. K. Knudsen.

Amandinea punctata (Hoffm.) Coppins \& Scheid. [9]

West Frontier Mts, Mt. Vlahina, Blagoevgrad Province, SW of Padesh village, near a small riverbed, on red basalt, 41 ${ }^{\circ} 54^{\prime} 22^{\prime \prime} \mathrm{N} 22^{\circ} 57^{\prime} 50^{\prime \prime} \mathrm{E}$, alt. 655 m, 26 May 2010, leg. A. Atanassova $090301 \&$ H. Mayrhofer.

*Arthonia mediella Nyl. [14]

Pirin Mts, Blagoevgard Province, on Pinus peuce, 41 $45^{\prime} 48^{\prime \prime} \mathrm{N} 23^{\circ} 25^{\prime} 13^{\prime \prime} \mathrm{E}$, alt. 1875 m, 10 Jul 2007, leg. H. Mayrhofer 18634 \& S. Nikolova. 
\#Aspicilia hoffmanniana (S. Ekman \& Fröberg ex R. Sant.) Cl. Roux \& M. Bertrand [5] Published by Szatala (1929: 91, as 'Aspicilia hoffmannii and 'A. caesioalba'). Mayrhofer et al. (2005) treated this taxon as 'A. contorta subsp. hoffmanniana'.

Brodoa intestiniformis (Vill.) Goward [11]

Mt. Belasitsa, Blagoevgrad Province, Petrich, at Maluk Kongour Peak, on siliceous rock outcrops, 41 ${ }^{\circ} 19^{\prime} 48^{\prime \prime} \mathrm{N} 23^{\circ} 10^{\prime} 31^{\prime \prime} \mathrm{E}$, alt. 1839 m, 27 May 2010, leg. A. Atanassova 110902 \& H. Mayrhofer.

Buellia disciformis (Fr.) Mudd [5]

Balkan Range, Lovech Province, Troyan, Beklemeto Mountain pass, along the road, on

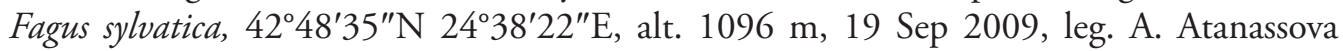
051409.

Buellia griseovirens (Turner \& Borrer ex Sm.) Almb. [14]

Pirin Mts, Blagoevgrad Province, trail to Vihren, on lignum of Pinus heldreichii, $41^{\circ} 46^{\prime} 22^{\prime \prime} \mathrm{N}$ 2325'35"E, alt. 2011 m, 11 Jul 2007, leg. H. Mayrhofer 18680 \& S. Nikolova (filed under Pycnora praestabilis).

\#Caloplaca arnoldii subsp. oblitterata (Pers.) Gaya [5, 17]

Published by Szatala (1930: 100, as 'Caloplaca pusilla var. obliterascens') and Gaya (2009: 45). Mayrhofer et al. (2005) treated this taxon as synonym of C. obliterans.

*Caloplaca asserigena (Stizenb. ex J. Lahm) Dalla Torre \& Sarnth. [17] The Rhodopes, Smolyan Province, above Trigrad Gorge, on Acerpseudoplatanus, 41 36'46"N 24²2'39"E, alt. 1200 m, 16 May 2008, leg. H. Mayrhofer 18574 \& S. Nikolova, det. J. Vondrák.

*Caloplaca atroflava (Turner) Mong. [17]

The Rhodopes, Kurdzhali Province, stonefield S of Stouden Kladenets village, on basalt, $41^{\circ} 36^{\prime} 10^{\prime \prime} \mathrm{N} 25^{\circ} 38^{\prime} 21^{\prime \prime} \mathrm{E}$, alt. 190 m, 14 May 2008, leg. H. Mayrhofer 18513 \& S. Nikolova, det. J. Vondrák.

Caloplaca demissa (Körb.) Arup \& Grube [12]

Mt. Slavyanka, Blagoevgrad Province, SW of Petrovo village, Valley of Petrovska Reka River, S-facing open dry rocky slopes, on mica schist, $41^{\circ} 25^{\prime} 29^{\prime \prime} \mathrm{N} 23^{\circ} 31^{\prime} 27^{\prime \prime} \mathrm{E}$, alt. $534 \mathrm{~m}$, 28 May 2010, leg. A. Atanassova 120106 \& H. Mayrhofer.

Caloplaca inconnexa (Nyl.) Zahlbr. var. inconnexa [18]

Thracian Lowland, Plovdiv Province, Besaparski Ridove, on limestone, 42 $5^{\prime} 13^{\prime \prime} \mathrm{N}$ 24²8'47"E, alt. 200 m, 13 May 2008, leg. H. Mayrhofer 18198 \& S. Nikolova, det. J. Vondrák. 
Caloplaca inconnexa var. nesodes Poelt \& Nimis [17]

The Rhodopes, Smolyan Province, Gela village, forest edge above pastures, on calcareous earthy boulders, $41^{\circ} 39^{\prime} 53^{\prime \prime} \mathrm{N} 24^{\circ} 34^{\prime} 5^{\prime \prime} \mathrm{E}$, alt. $1526 \mathrm{~m}$, 1 Aug 2009, leg. A. Atanassova 171105, det. J. Vondrák.

Caloplaca marmorata (Bagl.) Jatta [18]

Thracian Lowland, Plovdiv Province, Besaparski Ridove, on limestone, 42 ${ }^{\circ} 5^{\prime} 13^{\prime \prime} \mathrm{N}$ $24^{\circ} 28^{\prime} 47^{\prime \prime}$ E, alt. 220 m, 13 May 2008, leg. H. Mayrhofer 19713 \& S. Nikolova, det. J. Vondrák.

\#Caloplaca monacensis (Leder.) Lettau [17, 20]

Published by Szatala (1930: 97, as 'Caloplaca cerina f. cyanolepra'; Vondrák et al. 2009: 11; Šoun et al. 2011: 116). Mayrhofer et al. (2005) treated C. cerina f. cyanolepra as synonym of C. cerina.

\#Caloplaca pusilla (A. Massal.) Zahlbr. [5, 17]

Published by Szatala (1929: 97, as 'Caloplaca pusilla f. calcicola'; 1930: 100, as 'Caloplaca murorum var. pulvinatum'). Mayrhofer et al. (2005) treated these taxa as synonyms of $C$. saxicola.

\#Caloplaca rouxii Gaya, Nav.-Ros. \& Llimona [17]

Published by Szatala 1930: 99-100, as 'Caloplaca murorum var. miniatum'). Mayrhofer et al. (2005) treated this taxon as synonym of C. saxicola.

*Caloplaca subpallida H. Magn. [17]

The Rhodopes, Haskovo Province, along the road between Borislavtsi and Madzharovo, on trachyte, 41 39' $51^{\prime \prime} \mathrm{N} 25^{\circ} 54^{\prime} 6^{\prime \prime} \mathrm{E}$, alt. 220 m, 13 May 2008, leg. H. Mayrhofer 18162 \& S. Nikolova; near Madzharovo, steep N-facing slope, on basalt, 41 $38^{\prime} 24^{\prime \prime} \mathrm{N} 25^{\circ} 50^{\prime} 48^{\prime \prime} \mathrm{E}$, alt. 160 m, 14 May 2008, leg. H. Mayrhofer 18179 \& S. Nikolova, det. J. Vondrák.

Caloplaca variabilis (Pers.) Müll. Arg. [6, 18]

Sofia Region, Sofia Province, hills near Beledie Han, on calcareous outcrops, 42 $2^{\circ} 53^{\prime 2} 28^{\prime \prime} \mathrm{N}$ $23^{\circ} 9^{\prime} 48^{\prime} \mathrm{E}$, alt. $765 \mathrm{~m}, 15 \mathrm{Jul} 2007$, leg. H. Mayrhofer \& S. Nikolova (SOMF 26420); Thracian Lowland, Plovdiv Province, Besaparski Ridove, on limestone, 42 ${ }^{\circ} 5^{\prime 1} 3^{\prime \prime} \mathrm{N}$ $24^{\circ} 28^{\prime} 47^{\prime \prime E}$, alt. 200 m, 13 May 2008, leg. H. Mayrhofer 18160 \& S. Nikolova.

Candelariella reflexa (Nyl.) Lettau $[10,17,18]$

Valley of River Strouma, Blagoevgrad Province, Kresna Gorge, Tisata Nature Reserve,

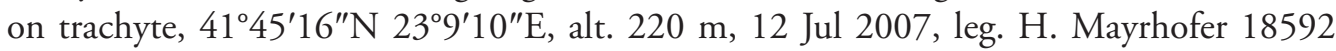
\& S. Nikolova; the Rhodopes, Smolyan Province, above Trigrad Gorge, on Juniperus

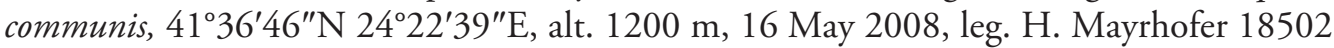
\& S. Nikolova; Thracian Lowland, Haskovo Province, Mineralni Bani village, Aida Peak,

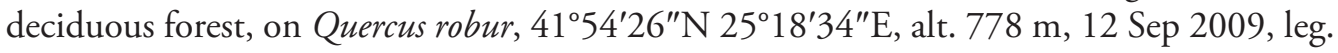


A. Atanassova 174606; Haskovo, Kenana, on Quercus frainetto, $41^{\circ} 57^{\prime} 32^{\prime \prime} \mathrm{N} 23^{\circ} 30^{\prime} 53^{\prime \prime} \mathrm{E}$, alt. 248 m, 28 Sep 2009, leg. A. Atanassova 180205.

Candelariella vitellina (Hoffm.) Müll. Arg. [9]

West Frontier Mts, Mt. Vlahina, Blagoevgrad Province, Padesh village, SE-facing pasture

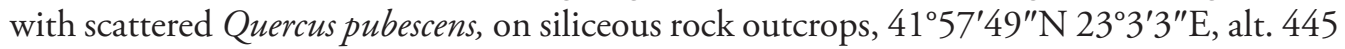
m, 26 May 2010, leg. A. Atanassova 090105 \& H. Mayrhofer.

* Catillaria detractula (Nyl.) H. Olivier [17]

The Rhodopes, Haskovo Province, Kralevo village, at Quercus forest edge, on hard calcareous earthy boulders, 41 $47^{\prime} 7^{\prime \prime} \mathrm{N} 25^{\circ} 40^{\prime} 49^{\prime \prime} \mathrm{E}$, alt. $280 \mathrm{~m}, 12$ Sep 2009, leg. A. Atanassova 174805 .

Catillaria lenticularis (Ach.) Th. Fr. [17]

The Rhodopes, Kurdzhali Province, the dam of Stouden Kladenets Reservoir, on trachyte, 41 36'59"N 2538'30"E, alt. 230 m, 14 May 2008, leg. H. Mayrhofer 18539 \& S. Nikolova.

Cetraria muricata (Ach.) Eckfeldt [11]

Mt. Belasitsa, Blagoevgrad Province, Kongour Peak, N-facing siliceous rock outcrops below

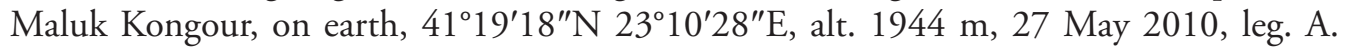
Atanassova 110705 \& H. Mayrhofer, det. Fernando Fernandez Mendoza.

Cladonia fimbriata (L.) Fr. [1, 9]

Black Sea coast, Bourgas Province, Sinemorets village, Silistar Protected Area, deciduous forest above the beach, on earth, 42 $2^{\prime} 13^{\prime \prime} \mathrm{N} 28^{\circ} 0^{\prime} 9^{\prime \prime} \mathrm{E}$, alt. $8 \mathrm{~m}, 23$ Sep 2009, leg. A. Atanassova 010603; West Frontier Mts, Mt. Vlahina, Blagoevgrad Province, Padesh village, beneath scattered Quercus cerris SW along the main road, on earth, $41^{\circ} 53^{\prime} 56^{\prime \prime} \mathrm{N} 22^{\circ} 56^{\prime} 52^{\prime \prime} \mathrm{E}$, alt. 830 m, 26 May 2010, leg. A. Atanassova 090225 \& H. Mayrhofer.

Cladonia symphycarpa (Flörke) Fr. [14]

Pirin Mts, Blagoevgrad Province, near Mountain Shelter Kazana, on soil, 41 46'12"N 2324'24"E, alt. 2408 m, 11 Jul 2007, leg. H. Mayrhofer 18646 \& S. Nikolova.

Collema flaccidum (Ach.) Ach. [11]

Mt. Belasitsa, Blagoevgrad Province, Kongour Hut, beech forest, on Fagus sylvatica, $41^{\circ} 20^{\prime} 38^{\prime \prime N} 23^{\circ} 11^{\prime} 12^{\prime \prime E}$, alt. 1370 m, 27 May 2010, leg. A. Atanassova 110304 \& H. Mayrhofer.

Cornicularia normoerica (Gunnerus) Du Rietz [11]

Mt. Belasitsa, Blagoevgrad Province, Kongour Peak, on N-facing siliceous rock outcrops below Maluk Kongour, 41 $19^{\prime} 18^{\prime \prime} \mathrm{N} 23^{\circ} 10^{\prime} 28^{\prime \prime} \mathrm{E}$, alt. 1944 m, 27 May 2010, leg. A. Atanassova $110716 \&$ H. Mayrhofer. 
Dermatocarpon miniatum (L.) W. Mann [10]

Valley of River Strouma, Blagoevgrad Province, Kresna Gorge, on siliceous rocks, $41^{\circ} 45^{\prime} 48^{\prime \prime} \mathrm{N}$ $23^{\circ} 09^{\prime} 10^{\prime \prime E}$, alt. 220 m, 12 Jul 2007, leg. H. Mayrhofer \& S. Nikolova (SOMF 26442).

Diploschistes muscorum (Scop.) R. Sant. [9, 12]

West Frontier Mts, Mt. Vlahina, Blagoevgrad Province, Padesh village, SE-facing pasture with scattered Quercus pubescens, on siliceous rock outcrops, 41 $1^{\circ} 57^{\prime} 49^{\prime \prime} \mathrm{N} 23^{\circ} 3^{\prime} 3^{\prime \prime} \mathrm{E}$, alt. 445 m, 26 May 2010, leg. A. Atanassova 090104 \& H. Mayrhofer; Mt. Slavyanka, Blagoevgrad Province, SW of Petrovo village, Valley of Petrovska reka River, S-facing open dry rocky

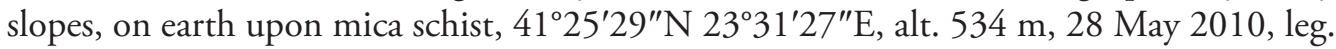
A. Atanassova $120113 \& \mathrm{H}$. Mayrhofer.

Diploschistes scruposus (Schreb.) Norman [12]

Mt. Slavyanka, Blagoevgrad Province, SW of Petrovo village, Valley of Petrovska Reka River, S-facing open dry rocky slopes, on mica schist, $41^{\circ} 25^{\prime} 29^{\prime \prime} \mathrm{N} 23^{\circ} 31^{\prime} 27^{\prime \prime} \mathrm{E}$, alt. $534 \mathrm{~m}$, 28 May 2010, leg. A. Atanassova 120105 \& H. Mayrhofer.

Diplotomma chlorophaeum (Hepp ex Leight.) Kr.P. Singh \& S.R. Singh [9, 10]

West Frontier Mts, Mt. Vlahina, Blagoevgrad Province, SW of Padesh village, near a

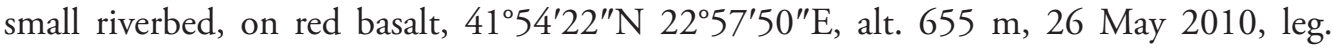
A. Atanassova 090302 \& H. Mayrhofer; Valley of River Strouma, Blagoevgrad Province, Kresna Gorge, Tisata Nature Reserve, on trachyte, $41^{\circ} 45^{\prime} 16^{\prime \prime} \mathrm{N} 23^{\circ} 9^{\prime} 10^{\prime \prime} \mathrm{E}$, alt. $220 \mathrm{~m}, 12$ Jul 2007, leg. H. Mayrhofer 18597 \& S. Nikolova.

*Diplotomma hedinii (H. Magn.) P. Clerc \& Cl. Roux $[6,12,17]$

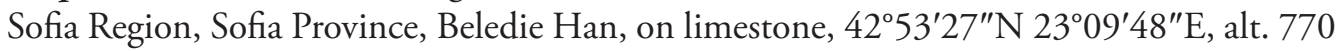
m, 15 July 2007, leg. H. Mayrhofer 18578 \& S. Nikolova; Mt. Slavyanka, Blagoevgrad Province, SW of Petrovo village, Valley of Petrovska Reka River, S-facing open dry rocky

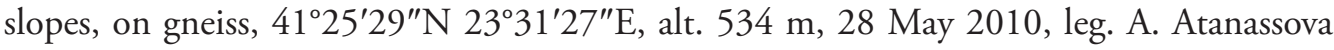
120126 \& H. Mayrhofer; the Rhodopes, Smolyan Province, Gela village, forest edge above pastures, on calcacerous earthy boulders, 41 $39^{\prime} 53^{\prime \prime} \mathrm{N} 24^{\circ} 34^{\prime} 5^{\prime \prime} \mathrm{E}$, alt. $1526 \mathrm{~m}, 1$ Aug 2009, leg. A. Atanassova 171102.

Diplotomma venustum (Körb.) Körb. [18]

Thracian Lowland, Plovdiv Province, Besaparski Ridove, on limestone, 42 ${ }^{\circ} 5^{\prime} 13^{\prime \prime} \mathrm{N}$ $24^{\circ} 28^{\prime} 47^{\prime \prime}$, alt. 200 m, 13 May 2008, leg. H. Mayrhofer 18197 \& S. Nikolova.

${ }^{*}$ Endohyalina insularis (Arnold) Giralt, van den Boom \& Elix [11, 14, 15] (syn. Rinodina insularis (Arnold) Hafellner)

Mt. Belasitsa, Blagoevgrad Province, Kongour Peak, N-facing siliceous rock outcrops below Maluk Kongour, on mica schist, lichenicolous on Lecanora rupicola, $41^{\circ} 19^{\prime} 18^{\prime \prime} \mathrm{N}$, $23^{\circ} 10^{\prime} 28^{\prime \prime}$ E, alt. 1944 m, 27 May 2010, leg. A. Atanassova 110722 \& H. Mayrhofer; Pirin Mts, Blagoevgrad Province, above Vihren Hut, near Okoto Lake, shaded siliceous rocks, 


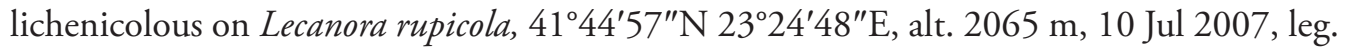
H. Mayrhofer \& S. Nikolova (SOMF 26440); Rila Mts, Kyustendil Province, free ascent to the Seven Rila Lakes, SW above Dolnoto Ezero Lake, exposed alpine meadow, on mica schist, lichenicolous on Lecanora rupicola, 42 $12^{\prime} 58^{\prime \prime} \mathrm{N} 23^{\circ} 19^{\prime} 13^{\prime \prime} \mathrm{E}$, alt. $2158 \mathrm{~m}, 25$ May 2010, leg. A. Atanassova 151004 \& H. Mayrhofer.

\# Graphis pulverulenta (Pers.) Ach. [15, 17]

Published by Suza (1929: 9, as 'Graphis scripta var. pulverulenta') and Popnikolov \& Zhelezova (1964: 108, as 'G. scripta var. abietina' and 'G. scripta var. pulverulenta'). Mayrhofer et al. (2005) treated these taxa as synonyms of $G$. scripta.

Gyalolechia flavovirescens (Wulfen) Søchting, Frödén \& Arup [5] (syn. Caloplaca flavovirescens (Wulfen) Dalla Torre \& Sarnth.)

Balkan Range, Mt. Ponor, Sofia Province, Dobravitsa village, Topilata neighbourhood, on

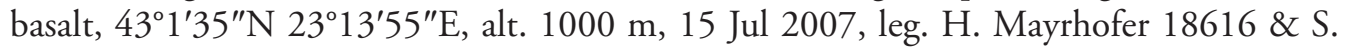
Nikolova.

Hypogymnia physodes (L.) Nyl. [11]

Mt. Belasitsa, Blagoevgrad Province, Kongour Peak, N-facing siliceous rock outcrops below Maluk Kongour, on dwarf shrups, $41^{\circ} 19^{\prime} 18^{\prime \prime} \mathrm{N} 23^{\circ} 10^{\prime} 28^{\prime \prime} \mathrm{E}$, alt. 1944 m, 27 May 2010, leg. A. Atanassova $110704 \& \mathrm{H}$. Mayrhofer.

Lambiella insularis (Nyl.) T. Sprib. [11, 14] (syn. Rimularia insularis (Nyl.) Rambold \& Hertel)

Mt. Belasitsa, Blagoevgrad Province, Kongour Peak, on N-facing siliceous rock outcrops below Maluk Kongour, lichenicolous on Lecanora rupicola, $41^{\circ} 20^{\prime} 3^{\prime \prime} \mathrm{N} 23^{\circ} 10^{\prime} 39^{\prime \prime} \mathrm{E}$, alt. 1698 m, 27 May 2010, leg. A. Atanassova 110608 \& H. Mayrhofer; Pirin Mts, Blagoevgrad Province, above Vihren Hut, near Okoto Lake, shaded siliceous rocks, lichenicolous on Lecanora rupicola, 41 $44^{\prime} 57^{\prime \prime N} 23^{\circ} 24^{\prime} 48^{\prime \prime E}$, alt. 2065 m, 10 Jul 2007, leg. H. Mayrhofer \& S. Nikolova (SOMF 26439).

Lecanora bicincta Ramond [10]

Valley of River Strouma, Blagoevgrad Province, W of Purvomay village, NW-facing open dry rocky slope, on mica schist, $41^{\circ} 24^{\prime} 10^{\prime \prime} \mathrm{N} 23^{\circ} 6^{\prime} 48^{\prime \prime} \mathrm{E}$, alt. 185 m, 29 May 2010, leg. A. Atanassova 100709 \& H. Mayrhofer.

Lecanora chlarotera Nyl. [9, 15, 17]

West Frontier Mts, Mt. Vlahina, Blagoevgrad Province, Padesh village, SE-facing pasture with scattered Quercus pubescens, 41 ${ }^{\circ} 57^{\prime} 49^{\prime \prime} \mathrm{N} 23^{\circ} 3^{\prime} 3^{\prime \prime} \mathrm{E}$, alt. 445 m, 26 May 2010, leg. A. Atanassova 090119 \& H. Mayrhofer; same locality, on Fraxinus ornus, leg. A. Atanassova 090125 \& H. Mayrhofer; Rila Mts, Kyustendil Province, Panichishte Resort, Populus plantation along the road, on Populus alba, 42 $16^{\prime} 57^{\prime \prime} \mathrm{N} 23^{\circ} 16^{\prime} 15^{\prime \prime} \mathrm{E}$, alt. $875 \mathrm{~m}, 24$ May 2010, leg. A. Atanassova 150116 \& H. Mayrhofer; the Rhodopes, Haskovo Province, Malko 
Gradishte village, cemetery with solitary trees, on Quercus robur, $41^{\circ} 46^{\prime} 2^{\prime \prime} \mathrm{N} 25^{\circ} 59^{\prime} 13^{\prime \prime} \mathrm{E}$, alt. 195 m, 23 Jul 2009, leg. A. Atanassova 170211.

\section{Lecanora gangaleoides $\mathrm{Nyl}$. [1]}

Published by Poelt \& Vězda (1981: 180), without locality.

Black Sea coast, Bourgas Province, Sinemorets village, on volcanic rock, $42^{\circ} 02^{\prime} 59^{\prime \prime} \mathrm{N}$ 2759'26"E, alt. 3 m, 15 Aug 2011, leg. A. Atanassova 011011, det. J. Malíček.

Lecanora intumescens (Rebent.) Rabenh. [20]

Mt. Strandzha, Bourgas Province, Varovnik village, roadside near the village, on Quercus

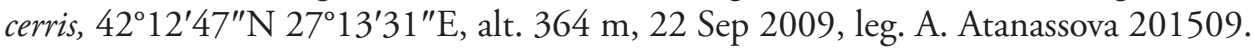

Lecanora mughicola Nyl. [8]

Vitosha Region, Sofia City, near Aleko Hut, on Pinus sylvestris, $42^{\circ} 34^{\prime} 18^{\prime \prime} \mathrm{N} 23^{\circ} 18^{\prime} 2^{\prime \prime} \mathrm{E}$, alt. 1868 m, 9 Jul 2007, leg. H. Mayrhofer \& S. Nikolova (SOMF 26476).

*Lecanora rouxii S. Ekman \& Tønsberg [5]

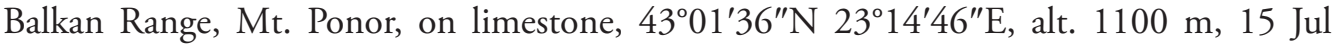
2007, leg. H. Mayrhofer 18608 \& S. Nikolova.

* Lecanora rupicola subsp. subplanata (Nyl.) Leuckert \& Poelt [11]

Mt. Belasitsa, Blagoevgrad Province, along the marked path from Kongour Hut to Kongour Peak just below Maluk Kongour, on N-facing siliceous rocks, $41^{\circ} 20^{\prime} 03^{\prime \prime} \mathrm{N} 23^{\circ} 10^{\prime} 39^{\prime \prime} \mathrm{E}$, alt. 1698 m, 27 May 2010, leg. A. Atanassova 110606 \& H. Mayrhofer.

TLC: atranorin, roccellic acid, xanthones.

Lecanora saligna (Schrad.) Zahlbr. [9]

West Frontier Mts, Mt. Vlahina, Blagoevgrad Province, Padesh village, scattered deciduous trees SW along the main road, on Prunus padus, $41^{\circ} 53^{\prime} 56^{\prime \prime} \mathrm{N} 22^{\circ} 56^{\prime} 52^{\prime \prime} \mathrm{E}$, alt. $830 \mathrm{~m}, 26$ May 2010, leg. A. Atanassova 090210 \& H. Mayrhofer.

Lecanora sulphurea (Hoffm.) Ach. [12]

Mt. Slavyanka, Blagoevgrad Province, SW of Petrovo village, Valley of Petrovska Reka River, S-facing open dry rocky slopes, on mica schist, $41^{\circ} 25^{\prime} 29^{\prime \prime} \mathrm{N} 23^{\circ} 31^{\prime} 27^{\prime \prime} \mathrm{E}$, alt. $534 \mathrm{~m}$, 28 May 2010, leg. A. Atanassova 120108 \& H. Mayrhofer.

Lecanora swartzii (Ach.) Ach. [17]

The Rhodopes, Haskovo Province, near Madzharovo, steep N-facing slope, on basalt, $41^{\circ} 38^{\prime} 24^{\prime \prime} \mathrm{N} 25^{\circ} 50^{\prime} 48^{\prime \prime} \mathrm{E}$, alt. 160 m, 14 May 2008, leg. H. Mayrhofer 18183 \& S. Nikolova.

Lecanora varia (Hoffm.) Ach. [8]

Vitosha Region, Sofia City, near Aleko Hut, on Pinus sylvestris, $42^{\circ} 34^{\prime} 18^{\prime \prime} \mathrm{N} 23^{\circ} 18^{\prime} 2^{\prime \prime} \mathrm{E}$, alt. 1868 m, 9 Jul 2007, leg. H. Mayrhofer \& S. Nikolova (SOMF 26477). 
${ }^{*}$ Lecidea berengeriana (A. Massal.) Nyl. [14]

Pirin Mts, Blagoevgrad Province, near Mountain Shelter Kazana, on soil and plant debris, $41^{\circ} 46^{\prime} 12^{\prime \prime} \mathrm{N} 23^{\circ} 24^{\prime} 24^{\prime \prime}$ E, alt. 2408 m, 11 Jul 2007, leg. H. Mayrhofer 18689 \& S. Nikolova.

\#Lecidea grisella Flörke [17]

Published by Szatala (1930: 71). Mayrhofer et al. (2005) treated this taxon as synonym of Lecidea fuscoatra.

*Lecidea sarcogynoides Körb. [9]

West Frontier Mts, Mt. Vlahina, Blagoevgrad Province, Padesh village, SE-facing pasture with scattered Quercus pubescens, on siliceous rock outcrops, 41 $1^{\circ} 57^{\prime} 49^{\prime \prime} \mathrm{N} 23^{\circ} 3^{\prime} 3^{\prime \prime} \mathrm{E}$, alt. 445 m, 26 May 2010, leg. A. Atanassova 090103 \& H. Mayrhofer.

The record of Szatala (1930: 73), also cited by Hertel (2006: 46), refers to Lecidella stigmatea, according to Hertel (1971: 256).

Lecidella asema (Nyl.) Knoph \& Hertel [17]

The Rhodopes, Kurdzhali Province, the dam of Stouden Kladenets Reservoir, on trachyte, $41^{\circ} 36^{\prime} 59^{\prime \prime} \mathrm{N} 25^{\circ} 38^{\prime} 30^{\prime \prime}$, alt. 230 m, 14 May 2008, leg. H. Mayrhofer 18549 \& S. Nikolova.

Lepra amara (Ach.) Hafellner [10] (syn. Pertusaria amara (Axch.) Nyl.)

Valley of River Strouma, Blagoevgrad Province, Kresna Gorge, Tisata Nature Reserve, on trachyte, $41^{\circ} 45^{\prime} 16^{\prime \prime} \mathrm{N} 23^{\circ} 9^{\prime} 10^{\prime \prime} \mathrm{E}$, alt. $220 \mathrm{~m}, 12 \mathrm{Jul} 2007$, leg. H. Mayrhofer 18600 \& S. Nikolova.

${ }^{*}$ Lepra leucosora (Nyl.) Hafellner [17] (syn. Pertusaria leucosora Nyl.)

The Rhodopes, Haskovo Province, near Madzharovo, steep N-facing slope, on basalt, $41^{\circ} 38^{\prime} 24^{\prime \prime} \mathrm{N} 25^{\circ} 50^{\prime} 48^{\prime \prime}$, alt. 160 m, 14 May 2008, leg. H. Mayrhofer 18182 \& S. Nikolova.

${ }^{*}$ Lepraria borealis Loht. \& Tønsberg $[11,14]$

Mt. Belasitsa, Blagoevgrad Province, Kongour Peak, on N-facing siliceous rock outcrops

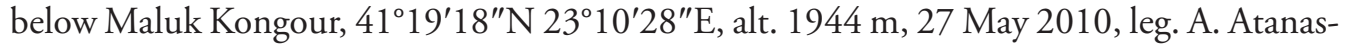
sova 110708a \& H. Mayrhofer; Pirin Mts, Blagoevgrad Province, along the trail to Vihren, on bryophytes, $41^{\circ} 44^{\prime} 56^{\prime \prime} \mathrm{N} 23^{\circ} 24^{\prime} 48^{\prime \prime} \mathrm{E}$, alt. $2031 \mathrm{~m}, 10 \mathrm{Jul}$ 2007, leg. H. Mayrhofer 18667 \& S. Nikolova.

Lepraria caesioalba (B. de Lesd.) J.R. Laundon [14]

Pirin Mts, Blagoevgrad Province, roadside between Bansko and Bunderitsa Hut, on bryophytes, $41^{\circ} 46^{\prime} 35^{\prime \prime} \mathrm{N} 23^{\circ} 26^{\prime} 21^{\prime \prime} \mathrm{E}$, alt. $1751 \mathrm{~m}$, 9 Jul 2007, leg. H. Mayrhofer 18683 \& S. Nikolova.

TLC: atranorin, fumarprotocetraric and roccellic acids. 
${ }^{*}$ Lepraria diffusa (J.R. Laundon) Kukwa [14, 17]

Pirin Mts, Blagoevgrad Province, below Vihren, on soil, $41^{\circ} 16^{\prime} 13^{\prime \prime} \mathrm{N} 23^{\circ} 24^{\prime} 40^{\prime \prime} \mathrm{E}$, alt. 2408 m, 11 Jul 2007, leg. H. Mayrhofer 18677 \& S. Nikolova; the Rhodopes, Smolyan Province, roadside between Chepelare and Pamporovo resort, on bryophytes, $41^{\circ} 39^{\prime} 31^{\prime \prime} \mathrm{N}$ $24^{\circ} 42^{\prime} 15^{\prime \prime}$ E, alt. 1500 m, 16 May 2008, leg. H. Mayrhofer $18557 \&$ S. Nikolova.

${ }^{*}$ Lepraria elobata Tønsberg $[8,15]$

Vitosha Region, Sofia City, near Aleko Hut, on Picea abies, 42 $34^{\prime} 16^{\prime \prime} \mathrm{N} 23^{\circ} 17^{\prime} 58^{\prime \prime} \mathrm{E}$, alt. 1838 m, 9 Jul 2007, leg. H. Mayrhofer 18681 \& S. Nikolova; Rila Mts, Kyustendil Province, free ascent to the Seven Rila Lakes, Picea abies forest, on fallen trunk, $42^{\circ} 13^{\prime} 56^{\prime \prime} \mathrm{N}$ $23^{\circ} 19^{\prime} 24^{\prime \prime}$ E, alt. 1903 m, 25 May 2010, leg. A. Atanassova 150502 \& H. Mayrhofer.

Lepraria finkii (B. de Lesd.) R.C. Harris $[8,17]$ (syn. L. lobificans Nyl.)

Vitosha Region, Sofia City, near Aleko Hut, on soil, 42 $34^{\prime} 16^{\prime \prime} \mathrm{N} 23^{\circ} 17^{\prime} 58^{\prime \prime} \mathrm{E}$, alt. 1838 m, 9 Jul 2007, leg. H. Mayrhofer 18682 \& S. Nikolova; the Rhodopes, Smolyan Province,

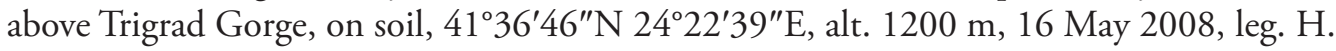
Mayrhofer 18573 \& S. Nikolova.

Lepraria nivalis J.R. Laundon [10] (syn. L. crassissima auct.)

Valley of River Strouma, Blagoevgrad Province, Roupite near Petrich, on steep W-facing volcanic rocks above the man-made stone cross, on trachyte, $41^{\circ} 27^{\prime} 32^{\prime \prime} \mathrm{N} 23^{\circ} 15^{\prime} 42^{\prime \prime} \mathrm{E}$, alt. 100 m, 26 May 2010, leg. A. Atanassova 100211 \& H. Mayrhofer; on huge sandstone tomb near the Strouma River bridge, 41 $29^{\prime} 17^{\prime \prime N} 23^{\circ} 15^{\prime} 35^{\prime \prime}$ E, alt. 110 m, 26 May 2010, leg. A. Atanassova 1003015 \& H. Mayrhofer.

* Lepraria nylanderiana Kümmerl. \& Leuckert [10]

Valley of River Strouma, Blagoevgrad Province, Kresna Gorge, Tisata Nature Reserve, on

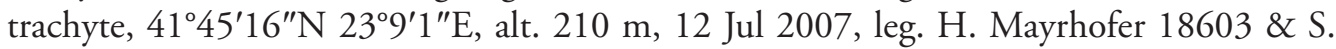
Nikolova.

${ }^{*}$ Lepraria vouauxii (Hue) R.C. Harris [17]

The Rhodopes, Haskovo Province, Kromleh near Dolni Glavanak village, on Quercus robur, 41 $40^{\prime} 54^{\prime \prime} \mathrm{N} 25^{\circ} 48^{\prime} 46^{\prime \prime}$ E, alt. 310 m, 15 May 2008, leg. H. Mayrhofer 18524 \& S. Nikolova.

Megaspora verrucosa (Ach.) Hafellner \& V. Wirth [15]

Rila Mts, Kyustendil Province, above Rilski Ezera Hut, NE-facing rocky slope, on moss upon amphibolite, $42^{\circ} 13^{\prime} 13^{\prime \prime} \mathrm{N} 23^{\circ} 19^{\prime} 12^{\prime \prime} \mathrm{E}$, alt. $2140 \mathrm{~m}, 25$ May 2010, leg. A. Atanassova 150804 \& H. Mayrhofer. 
Melanelia stygia (L.) Essl. [11]

Mt. Belasitsa, Blagoevgrad Province, Kongour Peak, on N-facing siliceous rock outcrops below Maluk Kongour, 41 $1^{\circ} 19^{\prime} 18^{\prime \prime} \mathrm{N} 23^{\circ} 10^{\prime} 28^{\prime \prime}$ E, alt. 1944 m, 27 May 2010, leg. A. Atanassova $110712 \&$ H. Mayrhofer (filed under Protoparmelia badia).

Melanelixia glabratula (Lamy) Sandler \& Arup [10, 17, 19] (syn. Melanelia fuliginosa subsp. glabratula (Lamy) Coppins)

Valley of River Strouma, Blagoevgrad Province, Kresna Gorge, Tisata Nature Reserve, on

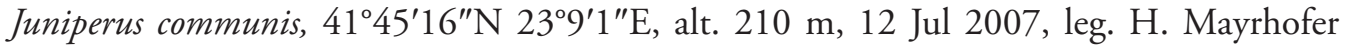
18581 \& S. Nikolova; the Rhodopes, Pazardzhik Province, Yundola village, Haidushka

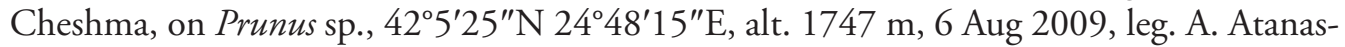
sova 171507; Haskovo Province, Kralevo village, deciduous forest, on Prunus sp., $41^{\circ} 47^{\prime} 2^{\prime \prime} \mathrm{N}$ 2540'55"E, alt. 330 m, 12 Sep 2009, leg. A. Atanassova 175102; Toundzha Hilly Country, Bourgas Province, Sredets, Malka Reka eco-path, deciduous forest, on fence out of young

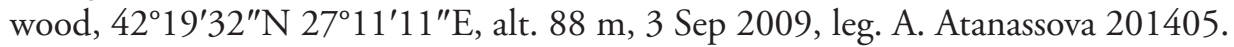

Melanelixia subargentifera (Nyl.) O. Blanco et al. $[10,12]$ (syn. Melanelia subargentifera (Nyl.) Essl.)

Valley of River Strouma, Blagoevgrad Province, Kresna Gorge, Tisata Nature Reserve, on Juniperus communis, $41^{\circ} 45^{\prime} 16^{\prime \prime} \mathrm{N} 23^{\circ} 9^{\prime} 1^{\prime \prime} \mathrm{E}$, alt. $210 \mathrm{~m}, 12 \mathrm{Jul}$ 2007, leg. H. Mayrhofer 18583 \& S. Nikolova; Mt. Slavyanka, Blagoevgrad Province, Izvora Hut, SW of Petrovo village, on Fraxinus ornus, 41 $24^{\prime} 53^{\prime \prime} \mathrm{N} 23^{\circ} 33^{\prime} 27^{\prime \prime}$, alt. 707 m, 28 May 2010, leg. A. Atanassova $120306 \&$ H. Mayrhofer.

Melanelixia subaurifera (Nyl.) O. Blanco et al. [10] (syn. Melanelia subaurifera (Nyl.) Essl.) Valley of River Strouma, Blagoevgrad Province, W of Purvomay village, NW-facing open dry rocky slope with shrubs destructed by grazing stock, on Carpinus orientalis, $41^{\circ} 24^{\prime} 10^{\prime \prime} \mathrm{N}$ $23^{\circ} 6^{\prime} 48^{\prime \prime} \mathrm{E}$, alt. 185 m, 29 May 2010, leg. A. Atanassova 100729 \& H. Mayrhofer.

Melanohalea exasperata (De Not.) O. Blanco et al. [12] (syn. Melanelia exasperata (De Not.) Essl.)

Mt. Slavyanka, Blagoevgrad Province, Izvora Hut, SW of Petrovo village, on Fraxinus ornus, $41^{\circ} 24^{\prime} 53^{\prime \prime} \mathrm{N} 23^{\circ} 33^{\prime} 27^{\prime \prime} \mathrm{E}$, alt. 707 m, 28 May 2010, leg. A. Atanassova 120319 \& H. Mayrhofer.

Miriquidica invadens Hafellner, Obermayer \& Tretiach [14]

Pirin Mts, Blagoevgrad Province, above Vihren Hut, on Sporastatia polyspora on granite, $41^{\circ} 44^{\prime} 56^{\prime \prime} \mathrm{N} 23^{\circ} 24^{\prime} 48^{\prime \prime} \mathrm{E}$, alt. $2031 \mathrm{~m}, 10 \mathrm{Jul}$ 2007, leg. H. Mayrhofer 18665 \& S. Nikolova, det. J. Hafellner.

TLC: miriquidic and stictic acids. 
Monerolechia badia (Fr.) Kalb [9, 10, 17] (syn. Buellia badia (Fr.) A. Massal.)

West Frontier Mts, Mt. Vlahina, Blagoevgrad Province, Padesh village, SE-facing pasture with scattered Quercus pubescens, on siliceous rock outcrops, $41^{\circ} 57^{\prime} 49^{\prime \prime} \mathrm{N} 23^{\circ} 3^{\prime} 3^{\prime \prime} \mathrm{E}$, alt. 445 m, 26 May 2010, leg. A. Atanassova 090102 \& H. Mayrhofer; Valley of River Strouma, Blagoevgrad Province, Roupite, on steep W-facing volcanic rocks above the man-made stone cross, on trachyte, $41^{\circ} 27^{\prime} 32^{\prime \prime} \mathrm{N} 23^{\circ} 15^{\prime} 42^{\prime \prime} \mathrm{E}$, alt. 100 m, 26 May 2010, leg. A. Atanassova 100202 \& H. Mayrhofer; the Rhodopes, Haskovo Province, near Madzharovo, steep

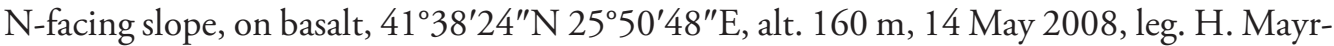
hofer 18184 \& S. Nikolova; Kurdzhali Province, the dam of Stouden Kladenets Reservoir,

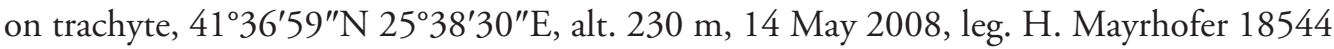
$\&$ S. Nikolova.

Myriolecis reuteri (Schaer.) Śliva, Zhao Xin \& Lumbsch [5] (syn. Lecanora reuteri Schaer.) Balkan Range, Mt. Ponor, Sofia Province, Dobravitsa village, Topilata neighbourhood, on

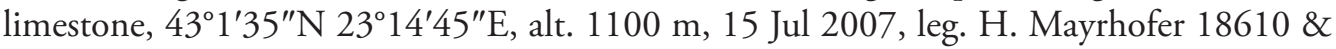
S. Nikolova.

Myriolecis sambuci (Pers.) Clem. [17] (syn. Lecanora sambuci (Pers.) Nyl.)

The Rhodopes, Haskovo Province, Malko Gradishte village, cemetery with solitary trees, on Juglans regia, 41 46'2"N 2559'13"E, alt. 195 m, 23 Jul 2009, leg. A. Atanassova 170207.

* Ochrolechia arborea (Kreyer) Almb. [17]

The Rhodopes, Smolyan Province, above Trigrad Gorge, on Juniperus communis, 41 36 '46”N $24^{\circ} 22^{\prime} 39^{\prime \prime}$, alt. 1200 m, 16 May 2008, leg. H. Mayrhofer 18508 \& S. Nikolova.

Parmelia submontana Nádv. ex Hale [9]

West Frontier Mts, Mt. Vlahina, Blagoevgrad Province, Padesh village, beneath scattered Quercus cerris SW along the main road, on earth, 41 $53^{\prime} 56^{\prime \prime} \mathrm{N} 22^{\circ} 56^{\prime} 52^{\prime \prime} \mathrm{E}$, alt. $830 \mathrm{~m}, 26$ May 2010, leg. A. Atanassova 090212 \& H. Mayrhofer.

Parmelina pastillifera (Harm.) Hale [9, 12, 15]

West Frontier Mts, Mt. Vlahina, Blagoevgrad Province, Padesh village, scattered deciduous trees SW along the main road, on Prunus padus, 41 ${ }^{\circ} 53^{\prime} 56^{\prime \prime} \mathrm{N} 22^{\circ} 56^{\prime} 52^{\prime \prime} \mathrm{E}$, alt. $830 \mathrm{~m}, 26$ May 2010, leg. A. Atanassova 090206 \& H. Mayrhofer; Mt. Slavyanka, Blagoevgrad Province, SW of Petrovo village, Valley of Petrovska Reka River, S-facing open dry rocky slopes, on mica schist, 412 $5^{\prime} 29^{\prime \prime} \mathrm{N} 23^{\circ} 31^{\prime} 27^{\prime \prime} \mathrm{E}$, alt. 534 m, 28 May 2010, leg. A. Atanassova 120109 $\&$ H. Mayrhofer; Rila Mts, Kyustendil Province, Panichishte Resort, Populus plantation along the road, on Populus alba, 42 $16^{\prime} 57^{\prime \prime} \mathrm{N} 23^{\circ} 16^{\prime} 15^{\prime \prime} \mathrm{E}$, alt. 875 m, 24 May 2010, leg. A. Atanassova 150115 \& H. Mayrhofer.

Parmelina quercina (Willd.) Hale [9, 11, 12, 18, 19]

West Frontier Mts, Mt. Vlahina, Blagoevgrad Province, Padesh village, scattered deciduous

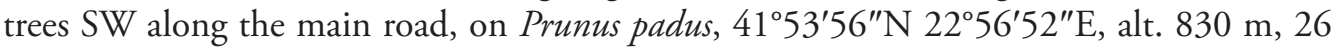


May 2010, leg. A. Atanassova 090207 \& H. Mayrhofer; Mt. Belasitsa, Blagoevgrad Province,

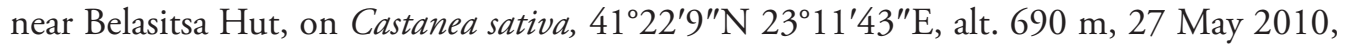
leg. A. Atanassova 110103 \& H. Mayrhofer; Mt. Slavyanka, Blagoevgrad Province, Izvora Hut, SW of Petrovo village, on Ailanthus altissima, 41 $24^{\prime} 53^{\prime \prime} \mathrm{N} 23^{\circ} 33^{\prime} 27^{\prime \prime} \mathrm{E}$, alt. $707 \mathrm{~m}$, 28 May 2010, leg. A. Atanassova 120318 \& H. Mayrhofer; Thracian Lowland, Haskovo Province, Haskovo, Kenana locality, on Quercus frainetto, $41^{\circ} 57^{\prime} 32^{\prime \prime} \mathrm{N} 25^{\circ} 30^{\prime} 53^{\prime \prime} \mathrm{E}$, alt. 248 m, 28 Sep 2009, leg. A. Atanassova 180207; Toundzha Hilly Country, Bourgas Province, Sredets, Malka Reka eco-path, deciduous forest, on Quercus robur, $42^{\circ} 19^{\prime} 47^{\prime \prime} \mathrm{N} 27^{\circ} 11^{\prime} 3^{\prime \prime} \mathrm{E}$, alt. 144 m, 3 Sep 2009, leg. A. Atanassova 201011.

Parmelina tiliacea (Hoffm.) Hale [9]

West Frontier Mts, Mt. Vlahina, Blagoevgrad Province, Padesh village, SE-facing pasture

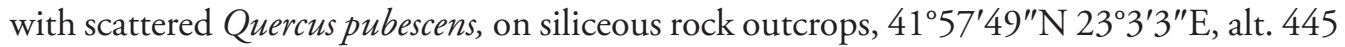
m, 26 May 2010, leg. A. Atanassova 090102 \& H. Mayrhofer.

Parmeliopsis hyperopta (Ach.) Vain. [8]

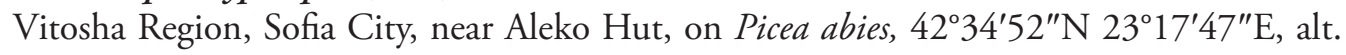
1838 m, 9 Jul 2007, leg. H. Mayrhofer \& S. Nikolova (SOMF 26503).

Peltigera elisabethae Gyeln. [1]

Black Sea coast, Bourgas Province, Sinemorets village, at a small beach, on earth above volcanic rocks, 422'59"N 2759'26"E, alt. 3 m, 15 Aug 2011, leg. A. Atanassova 011002, det. P. Resl.

Peltigera malacea (Ach.) Funck [11]

Mt. Belasitsa, Blagoevgrad Province, Kongour Peak, at Maluk Kongour Peak, on siliceous rock outcrops, 41 $19^{\prime} 48^{\prime \prime} \mathrm{N} 23^{\circ} 10^{\prime} 31^{\prime \prime} \mathrm{E}$, alt. 1839 m, 27 May 2010, leg. A. Atanassova $110901 \&$ H. Mayrhofer.

Peltigera membranacea (Ach.) Nyl. [17]

The Rhodopes, Smolyan Province, Smolyan, orchard around Ezerata Hut, on earth, $41^{\circ} 36^{\prime} 22^{\prime \prime N} 24^{\circ} 40^{\prime} 12^{\prime \prime} \mathrm{E}$, alt. 1373 m, 5 Sep 2009, leg. A. Atanassova 174205, det. P. Resl.

Peltigera praetextata (Flörke ex Sommerf.) Vain. [17]

The Rhodopes, Smolyan Province, Chepelare, Choudnite Mostove rock formation, eco-

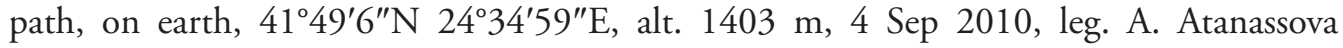
174008, det. P. Resl.

Peltigera rufescens (Weiss) Humb. [11]

Mt. Belasitsa, Blagoevgrad Province, Kongour Peak, just below Maluk Kongour Peak, on soil between bryophytes, 41 $19^{\prime} 18^{\prime \prime} \mathrm{N} 23^{\circ} 10^{\prime} 28^{\prime \prime} \mathrm{E}$, alt. $1944 \mathrm{~m}, 27$ May 2010, leg. A. Atanassova 110707 \& H. Mayrhofer. 


\section{*Pertusaria flavicans Lamy [17]}

The Rhodopes, Kurdzhali Province, the dam of Stouden Kladenets Reservoir, on trachyte, $4^{\circ} 36^{\prime} 59^{\prime \prime} \mathrm{N} 25^{\circ} 38^{\prime} 30^{\prime \prime}$ E, alt. 230 m, 14 May 2008, leg. H. Mayrhofer 18543 \& S. Nikolova.

Phlyctis argena (Ach.) Flot. [10]

Valley of River Strouma, Blagoevgrad Province, Purvomay village, Samouil's Fortress, on

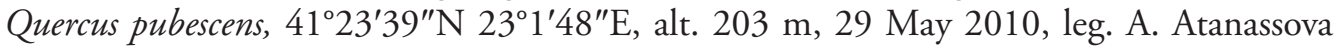
100507 \& H. Mayrhofer.

Physcia dubia (Hoffm.) Lettau [11]

Belasitsa mountain, Blagoevgrad Province, Maluk Kongour Peak, on siliceous rock outcrops, $41^{\circ} 19^{\prime} 48^{\prime \prime} \mathrm{N} 23^{\circ} 10^{\prime} 31^{\prime \prime} \mathrm{E}$, alt. $1839 \mathrm{~m}, 27$ May 2010, leg. A. Atanassova 110903 \& H. Mayrhofer, det. T.L. Esslinger.

Pleurosticta acetabulum (Neck.) Elix \& Lumbsch $[12,17]$

Mt. Slavyanka, Blagoevgrad Province, Izvora Hut, SW of Petrovo village, on Fraxinus ornus, 41 $24^{\prime} 53^{\prime \prime} \mathrm{N} 23^{\circ} 33^{\prime} 27^{\prime \prime} \mathrm{E}$, alt. 707 m, 28 May 2010, leg. A. Atanassova 120309 \& H. Mayrhofer; the Rhodopes, Haskovo Province, Malko Gradishte village, cemetery with solitary trees, on Juglans regia, 41 $46^{\prime} 2^{\prime \prime} \mathrm{N} 25^{\circ} 59^{\prime} 13^{\prime \prime} \mathrm{E}$, alt. $195 \mathrm{~m}$, $23 \mathrm{Jul} 2009$, leg. A. Atanassova 170205; Mineralni Bani village, Aida Peak, deciduous forest, on Quercus robur, $41^{\circ} 54^{\prime} 26^{\prime \prime} \mathrm{N} 25^{\circ} 18^{\prime} 34^{\prime \prime} \mathrm{E}$, alt. 778 m, 12 Sep 2009, leg. A. Atanassova 174605.

Porpidia cinereoatra (Ach.) Hertel \& Knoph [17]

The Rhodopes, Kurdzhali Province, stonefield $S$ of Stouden Kladenets village, on basalt, $41^{\circ} 36^{\prime} 10^{\prime \prime} \mathrm{N} 25^{\circ} 38^{\prime} 21^{\prime \prime} \mathrm{E}, 14$ May 2008, leg. H. Mayrhofer 18515 \& S. Nikolova, det. J. Malíček.

Porpidia crustulata (Zahlbr.) Hertel \& A. J. Schwab [11]

Mt. Belasitsa, Blagoevgrad Province, Kongour Peak, on silicate, $41^{\circ} 19^{\prime} 16^{\prime \prime} \mathrm{N} 23^{\circ} 10^{\prime} 29^{\prime \prime}$, alt. 1951 m, 27 May 2010, leg. A. Atanassova 110803 \& H. Mayrhofer.

Porpidia macrocarpa (DC.) Hertel \& A.J. Schwab [10, 12]

Valley of River Strouma, Blagoevgrad Province, Kresna Gorge, Tisata Nature Reserve, on trachyte, $41^{\circ} 45^{\prime} 16^{\prime \prime} \mathrm{N} 23^{\circ} 9^{\prime} 10^{\prime \prime} \mathrm{E}$, alt. $220 \mathrm{~m}, 12 \mathrm{Jul} 2007$, leg. H. Mayrhofer 18599 \& S. Nikolova; Mt. Slavyanka, Blagoevgrad Province, SW of Petrovo village, valley of Petrovska

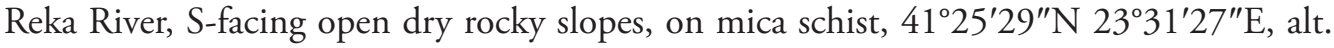
534 m, 28 May 2010, leg. A. Atanassova 120112 \& H. Mayrhofer.

Protoparmelia badia (Hoffm.) Hafellner [11]

Mt. Belasitsa, Blagoevgrad Province, Kongour Peak, on N-facing siliceous rock outcrops below Maluk Kongour, 41 ${ }^{\circ} 19^{\prime} 18^{\prime \prime} \mathrm{N} 23^{\circ} 10^{\prime} 28^{\prime \prime} \mathrm{E}$, alt. 1944 m, 27 May 2010, leg. A. Atanassova $110712 \& \mathrm{H}$. Mayrhofer. 
Protoparmelia montagnei (Fr.) Poelt \& Nimis [10, 12] (syn. P. psarophana (Nyl.) Sancho \& A. Crespo)

Valley of River Strouma, Blagoevgrad Province, Roupite, on huge sandstone tomb near the Strouma River bridge roadside to Roupite Monastery, 41 $29^{\prime} 17^{\prime \prime} \mathrm{N} 23^{\circ} 15^{\prime} 35^{\prime \prime} \mathrm{E}$, alt. $110 \mathrm{~m}$, 26 May 2010, leg. A. Atanassova 100306 \& H. Mayrhofer; Mt. Slavyanka, Blagoevgrad Province, SW of Petrovo village, valley of Petrovska Reka River, S-facing open dry rocky slopes, on mica schist, 41 $25^{\prime} 29^{\prime \prime} \mathrm{N} 23^{\circ} 31^{\prime} 27^{\prime \prime} \mathrm{E}$, alt. 534 m, 28 May 2010, leg. A. Atanassova 120115 \& H. Mayrhofer.

*Protoparmeliopsis muralis var. dubyi (Müll. Arg.) Hafellner \& Türk [10, 17] (syn. Lecanora muralis var. dubyi (Müll. Arg.) Poelt)

Valley of River Strouma, Blagoevgrad Province, Roupite, on huge sandstone tomb near

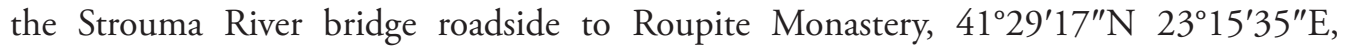
alt. 110 m, 26 May 2010, leg. A. Atanassova 100317 \& H. Mayrhofer; the Rhodopes, Haskovo Province, roadside between Borislavtsi and Madzharovo, on trachyte, $41^{\circ} 39^{\prime} 51^{\prime \prime} \mathrm{N}$ 2554'06"E, alt. 220 m, 13 May 2008, leg. H. Mayrhofer 18163 \& S. Nikolova.

\#Protoparmeliopsis versicolor (Pers.) M. Choisy [5, 17]

Published by Kasandzhiev (1900: 528, as 'Lecanora saxicola var. versicolor'), Suza (1929: 19, as 'L. saxicola var. versicolor'), and Szatala (1929: 94, 1930: 89, as 'Placodium murale var. versicolor'). Mayrhofer et al. (2005) treated these taxa as synonyms of Protoparmeliopsis muralis.

Psilolechia lucida (Ach.) M. Choisy [11]

Mt. Belasitsa, Blagoevgrad Province, Belasitsa Hut, Leshnishki Waterfall, deciduous forest, on earthy granitic outcrops, 41 $21^{\prime} 40^{\prime \prime} \mathrm{N} 23^{\circ} 10^{\prime} 57^{\prime \prime} \mathrm{E}$, alt. $780 \mathrm{~m}, 29$ May 2010, leg. A. Atanassova $111311 \&$ H. Mayrhofer.

* Pycnora praestabilis (Nyl.) Hafellner [14]

Pirin Mts, Blagoevgrad Province, near Bunderitsa Hut, on lignum of Pinus heldreichii, $41^{\circ} 46^{\prime} 03^{\prime \prime} \mathrm{N} 23^{\circ} 25^{\prime} 24^{\prime \prime}$, alt. 1883 m, 10 Jul 2007, H. Mayrhofer 18675 \& S. Nikolova; Blagoevgrad Province, trail to Vihren, on lignum of Pinus heldreichii, $41^{\circ} 46^{\prime} 22^{\prime \prime} \mathrm{N}$ $23^{\circ} 25^{\prime} 35^{\prime \prime}$ E, alt. $2011 \mathrm{~m}, 11 \mathrm{Jul}$ 2007, leg. H. Mayrhofer 18680 \& S. Nikolova (associated with Buellia griseovirens).

Ramalina fastigiata (Pers.) Ach. [10, 11, 17]

Valley of River Strouma, Blagoevgrad Province, Purvomay village, Samouil's Fortress, on Quercus pubescens, 41 23'39"N 231'48"E, alt. 203 m, 29 May 2010, leg. A. Atanassova 100508 \& H. Mayrhofer; Mt. Belasitsa, Blagoevgrad Province, near Belasitsa Hut, on Castanea sativa, 41 $22^{\prime} 9^{\prime \prime} \mathrm{N} 23^{\circ} 11^{\prime} 43^{\prime \prime} \mathrm{E}$, alt. $690 \mathrm{~m}, 27$ May 2010, leg. A. Atanassova 110104 \& H. Mayrhofer; the Rhodopes, Smolyan Province, near Trigrad, on Robinia pseuda-

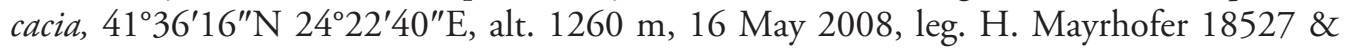


S. Nikolova; Chepelare, along the road to Pamporovo Resort, on Abies alba, $41^{\circ} 39^{\prime} 31^{\prime \prime} \mathrm{N}$ $24^{\circ} 42^{\prime} 15^{\prime \prime}$ E, alt. 1500 m, 16 May 2008, leg. H. Mayrhofer 18555 \& S. Nikolova.

Ramalina pollinaria (Westr.) Ach. [9, 10, 12]

West Frontier Mts, Mt. Vlahina, Blagoevgrad Province, Padesh village, scattered deciduous

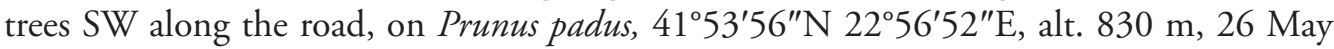
2010, leg. A. Atanassova 090214 \& H. Mayrhofer; Valley of River Strouma, Blagoevgrad Province, Roupite, on huge sandstone tomb near the Strouma River bridge, $41^{\circ} 29^{\prime} 17^{\prime \prime} \mathrm{N}$ $23^{\circ} 15^{\prime} 35^{\prime \prime}$ E, alt. 110 m, 26 May 2010, leg. A. Atanassova 100316 \& H. Mayrhofer; Mt. Slavyanka, Blagoevgrad Province, SW of Petrovo village, Valley of Petrovska Reka River,

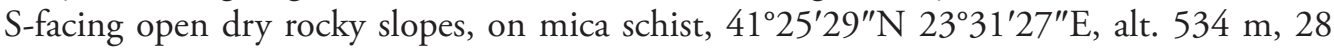
May 2010, leg. A. Atanassova $120111 \&$ H. Mayrhofer.

Rhizocarpon distinctum Th. Fr. [10, 11]

Valley of River Strouma, Blagoevgrad Province, Roupite, on huge sandstone tomb near the Strouma River bridge, $41^{\circ} 29^{\prime} 17^{\prime \prime N} 23^{\circ} 15^{\prime} 35^{\prime \prime}$, alt. 110 m, 26 May 2010, leg. A. Atanassova $100309 \& \mathrm{H}$. Mayrhofer; Purvomay village, Samouil's Fortress, NW-facing rock faces, on mica schist, $41^{\circ} 23^{\prime} 46^{\prime \prime} \mathrm{N} 23^{\circ} 1^{\prime} 36^{\prime \prime} \mathrm{E}$, alt. $185 \mathrm{~m}, 29$ May 2010, leg. A. Atanassova 100610 \& H. Mayrhofer; Mt. Belasitsa, Blagoevgrad Province, Kongour Peak, on N-facing siliceous rock outcrops below Maluk Kongour, 41 ${ }^{\circ} 19^{\prime} 18^{\prime \prime} \mathrm{N} 23^{\circ} 10^{\prime} 28^{\prime \prime} \mathrm{E}$, alt. $1944 \mathrm{~m}, 27$ May 2010, leg. A. Atanassova 110714 \& H. Mayrhofer.

\section{Rhizocarpon geminatum Körb. [15]}

Rila Mts, Kyustendil Province, above Rilski Ezera Hut, NE-facing rocky slope, on amphibolite, $42^{\circ} 13^{\prime} 13^{\prime \prime} \mathrm{N} 23^{\circ} 19^{\prime} 12^{\prime \prime} \mathrm{E}$, alt. $2140 \mathrm{~m}, 25$ May 2010, leg. A. Atanassova $150807 \&$ H. Mayrhofer.

Rhizocarpon reductum Th. Fr. [11]

Mt. Belasitsa, Blagoevgrad Province, Kongour Peak, on N-facing siliceous rock outcrops

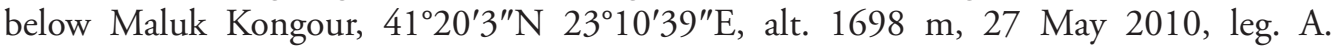
Atanassova 110605 \& H. Mayrhofer.

Rinodina bischoffii (Hepp) A. Massal. [5]

Balkan Range, Mt. Ponor, Sofia Province, Dobravitsa village, Topilata neighbourhood,

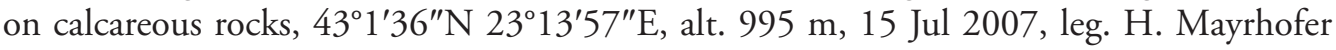
$18585 \&$ S. Nikolova (GZU, SOMF 26444).

Rinodina calcarea (Hepp ex Arnold) Arnold [18]

Thracian Lowland, Plovdiv Province, Besaparski Ridove, on limestone, 42 ${ }^{\circ} 5^{\prime} 13^{\prime \prime} \mathrm{N}$ $24^{\circ} 28^{\prime} 47^{\prime \prime E}$, alt. 200 m, 13 May 2008, leg. H. Mayrhofer 18195 \& S. Nikolova. 


\section{Rinodina capensis Hampe [17]}

The Rhodopes, Smolyan Province, above Trigrad Gorge, on Acerpseudoplatanus, 41 $36^{\prime} 46^{\prime \prime} \mathrm{N}$ $24^{\circ} 22^{\prime} 39^{\prime \prime}$ E, alt. 1200 m, 16 May 2008, leg. H. Mayrhofer 18576 \& S. Nikolova.

* Rinodina freyi H. Magn. [11, 17]

Mt. Belasitsa, Blagoevgrad Province, Kongour Peak, N-facing siliceous rock outcrops below Maluk Kongour, 41 $19^{\prime} 18^{\prime \prime} \mathrm{N} 23^{\circ} 10^{\prime} 28^{\prime \prime} \mathrm{E}$, alt. 1944 m, 27 May 2010, leg. A. Atanassova $110701 \&$ H. Mayrhofer; the Rhodopes, Smolyan Province, above Trigrad Gorge, on

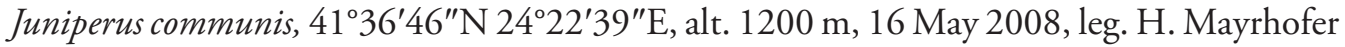
18503 \& S. Nikolova; Haskovo Province, Malko Gradishte village, cemetery with solitary trees, on Quercus robur, 41 46'2"N 2559'13"E, alt. 195 m, 23 Jul 2009, leg. A. Atanassova 170210.

Rinodina immersa (Körb.) J. Steiner [6]

Sofia Region, Sofia Province, Beledie Han, on calcareous outcrops, 42 $53^{\prime} 28^{\prime \prime} \mathrm{N} 23^{\circ} 9^{\prime} 48^{\prime \prime} \mathrm{E}$, alt. 765 m, 15 Jul 2007, leg. H. Mayrhofer \& S. Nikolova (SOMF 26476).

Rinodina lecanorina (A. Massal.) A. Massal. [5, 17]

Balkan Range, Mt. Ponor, Sofia Province, Dobravitsa village, Topilata neighbourhood, on limestone, 431'36"N 2313'56"E, alt. 1000 m, 15 Jul 2007, leg. H. Mayrhofer 18613

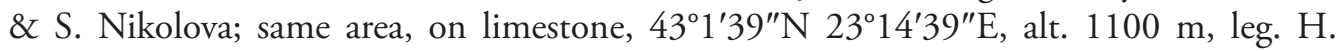
Mayrhofer 18624 \& S. Nikolova (with Rinodinella controversa); the Rhodopes, Smolyan Province, above Trigrad Gorge, on limestone, 41 $36^{\prime} 46^{\prime \prime} \mathrm{N} 24^{\circ} 22^{\prime} 39^{\prime \prime} \mathrm{E}$, alt. $1200 \mathrm{~m}, 16$ May 2008, leg. H. Mayrhofer 18506 \& S. Nikolova; same area, on breccia, 41 36'16"N $24^{\circ} 22^{\prime} 40^{\prime \prime}$ E, alt. 1260 m, 16 May 2008, leg. H. Mayrhofer 18529 \& S. Nikolova; eco-path along Trigrad Gorge, on calcareous vertical rocks, 41 $36^{\prime} 44^{\prime \prime} \mathrm{N} 24^{\circ} 22^{\prime} 47^{\prime \prime} \mathrm{E}$, alt. $1140 \mathrm{~m}, 25$ Jul 2010, leg. A. Atanassova 174406.

* Rinodina luridata var. immersa (H. Mayrhofer \& Cl. Roux) Cl. Roux [17]

The Rhodopes, Smolyan Province, Gela village, forest edge above pastures, on calcacerous

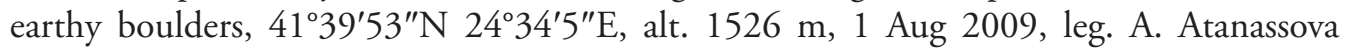
171107 (with Aspicilia calcarea).

Rinodina milvina (Wahlenb.) Th. Fr. [11]

Mt. Belasitsa, Blagoevgrad Province, Kongour Peak, on N-facing siliceous rock outcrops below Maluk Kongour, 41 $20^{\prime} 3^{\prime \prime} \mathrm{N} 23^{\circ} 10^{\prime} 39^{\prime \prime} \mathrm{E}$, alt. 1698 m, 27 May 2010, leg. A. Atanassova 110603 \& H. Mayrhofer; same area, on mica schist, $41^{\circ} 19^{\prime} 18^{\prime \prime} \mathrm{N} 23^{\circ} 10^{\prime} 28^{\prime \prime} \mathrm{E}$, alt. 1944 m, leg. A. Atanassova 110721 \& H. Mayrhofer.

Rinodina obnascens (Nyl.) H. Olivier [10]

Valley of River Strouma, Blagoevgrad Province, W of Purvomay village, NW-facing open dry rocky slope, on mica schist, lichenicolous on Aspicilia sp., sterile, $41^{\circ} 24^{\prime} 10^{\prime \prime} \mathrm{N}$ $23^{\circ} 6^{\prime} 48^{\prime \prime}$ E, alt. 185 m, 29 May 2010, leg. A. Atanassova 100706 \& H. Mayrhofer. 
*Rinodina occulta (Körb.) Sheard [10]

Valley of River Strouma, Blagoevgrad Province, W of Purvomay village, NW-facing open dry rocky slope, on mica schist, $41^{\circ} 24^{\prime} 10^{\prime \prime} \mathrm{N} 23^{\circ} 6^{\prime} 48^{\prime \prime} \mathrm{E}$, alt. $185 \mathrm{~m}, 29$ May 2010, leg. A. Atanassova 100705 \& H. Mayrhofer.

Rinodina oleae Bagl. [10] (syn. R. gennarii Bagl.)

Valley of River Strouma, Blagoevgrad Province, Kresna Gorge, Tisata Nature Reserve, on Juniperus communis, $41^{\circ} 45^{\prime} 16^{\prime \prime} \mathrm{N} 23^{\circ} 9^{\prime} 1^{\prime \prime} \mathrm{E}$, alt. $210 \mathrm{~m}, 12 \mathrm{Jul} 2007$, leg. H. Mayrhofer 18580 \& S. Nikolova; Purvomay village, Samouil's Fortress, NW-facing rock faces, on mica

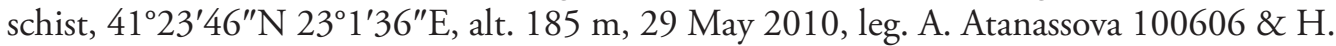
Mayrhofer.

Rinodina pyrina (Ach.) Arnold [5, 9, 12, 15, 19]

Balkan Range, Mt. Ponor, Sofia Province, Dobravitsa village, Topilata neighbourhood, on

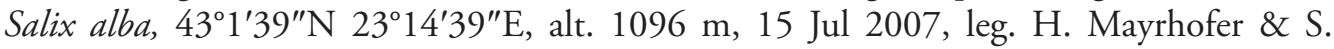
Nikolova (SOMF 26447); same locality, on Juglans regia, leg. H. Mayrhofer \& S. Nikolova (SOMF 26446); West Frontier Mts, Mt. Vlahina, Blagoevgrad Province, Padesh village, SEfacing pasture with scattered deciduous trees, on Quercus pubescens, $41^{\circ} 57^{\prime} 49^{\prime \prime} \mathrm{N} 23^{\circ} 3^{\prime} 3^{\prime \prime} \mathrm{E}$, alt. 445 m, 26 May 2010, leg. A. Atanassova 090115 \& H. Mayrhofer; same locality, on Fraxinus ornus, leg. A. Atanassova 090120 \& H. Mayrhofer; SW of Padesh village,

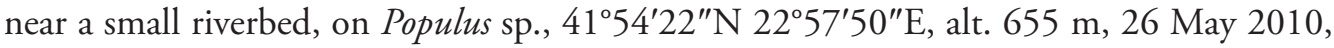
leg. A. Atanassova 090315 \& H. Mayrhofer; Mt. Slavyanka, Blagoevgrad Province, SW of Petrovo village, Valley of Petrovska Reka River, S-facing open dry rocky slopes, on Juniperus oxycedrus, $41^{\circ} 25^{\prime} 29^{\prime \prime} \mathrm{N} 23^{\circ} 31^{\prime} 27^{\prime \prime} \mathrm{E}$, alt. 534 m, 28 May 2010, leg. A. Atanassova 120130 \& H. Mayrhofer; $S$ of Goleshovo village, open pasture with scattered old deciduous trees, on Cornus mas, 41 24'25"N 2335'15"E, alt. 997 m, 28 May 2010, leg. A. Atanassova 120417 $\&$ H. Mayrhofer; Rila Mts, Kyustendil Province, Panichishte Resort, meadow along the road, on Juglans regia, $42^{\circ} 16^{\prime} 57^{\prime \prime} \mathrm{N} 23^{\circ} 16^{\prime} 15^{\prime \prime} \mathrm{E}$, alt. $875 \mathrm{~m}, 24$ May 2010, leg. A. Atanassova 150205 \& H. Mayrhofer; Toundzha Hilly Country, Bourgas Province, Sredets, Malka reka eco-path, deciduous forest, on fence out of young wood, $42^{\circ} 19^{\prime} 32^{\prime \prime} \mathrm{N} 27^{\circ} 11^{\prime} 11^{\prime \prime} \mathrm{E}$, alt. 88 m, 3 Sep 2009, leg. A. Atanassova 201401.

* Rinodina roscida (Sommerf.) Arnold [14]

Pirin Mts, Blagoevgrad Province, near Mountain Shelter Kazana, on soil and plant debris, $41^{\circ} 46^{\prime} 12^{\prime \prime} \mathrm{N} 23^{\circ} 24^{\prime} 24^{\prime \prime}$, alt. 2408 m, 11 Jul 2007, leg. H. Mayrhofer 18644 \& S. Nikolova.

* Rinodina sicula $\mathrm{H}$. Mayrhofer \& Poelt [1]

Black Sea coast, Bourgas Province, Sinemorets village, Lipite Beach, on volcanic rocks,

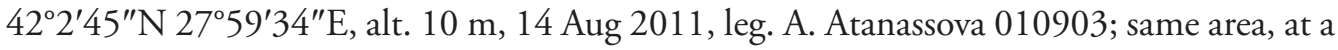
small beach along the eco-path, on volcanic rocks, $42^{\circ} 2^{\prime} 59^{\prime \prime} \mathrm{N} 27^{\circ} 59^{\prime} 26^{\prime \prime} \mathrm{E}$, alt. $3 \mathrm{~m}, 15$ Aug 2011, leg. A. Atanassova 011002; estuary of Veleka River, on volcanic rocks, $42^{\circ} 3^{\prime} 54^{\prime \prime} \mathrm{N}$ $27^{\circ} 58^{\prime} 33^{\prime \prime}$, alt. $10 \mathrm{~m}, 22$ Sep 2010, leg. A. Atanassova 010804.

First records from the Balkan Peninsula. 
Rinodina sophodes (Ach.) A. Massal. [1, 5, 9, 10, 11, 12]

Black Sea coast, Bourgas Province, Emona village, Cape Emine, pastures with solitary trees

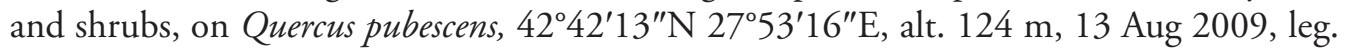
A. Atanassova 011207; Irakli, on Prunus dulcis, 42 $45^{\prime} 06^{\prime \prime} \mathrm{N} 27^{\circ} 53^{\prime} 19^{\prime \prime} \mathrm{E}$, alt. 8 m, 13 Aug 2009, leg. A. Atanassova 010108; Balkan Range, Mt. Ponor, Sofia Province, Dobravitsa

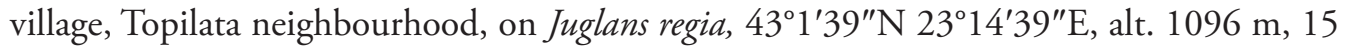
Jul 2007, leg. H. Mayrhofer \& S. Nikolova (SOMF 26453); Lovech Province, Beklemeto mountain pass, along the road, on Fagus sylvatica, 42 48'35"N $24^{\circ} 38^{\prime} 22^{\prime \prime} \mathrm{E}$, alt. $1096 \mathrm{~m}$, 19 Sep 2009, leg. A. Atanassova 051410; West Frontier Mts, Mt. Vlahina, Blagoevgrad Province, Padesh village, SE-facing pasture with scattered Quercus pubescens, 41 $57^{\prime} 49^{\prime \prime} \mathrm{N}$ 233'3"E, alt. 445 m, 26 May 2010, leg. A. Atanassova 090116 \& H. Mayrhofer; same area, SW of Padesh, scattered deciduous trees along the main road, on Prunus padus, 41 ${ }^{\circ} 53^{\prime} 56^{\prime \prime} \mathrm{N} 22^{\circ} 56^{\prime} 52^{\prime \prime} \mathrm{E}$, alt. 830 m, 26 May 2010, leg. A. Atanassova 090204 \& H. Mayrhofer; Valley of River Strouma, Blagoevgrad Province, Kresna Gorge, Tisata Nature

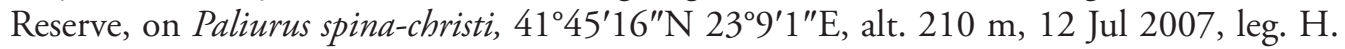
Mayrhofer \& S. Nikolova (SOMF 26465); Mt. Belasitsa, Blagoevgrad Province, Kongour Hut, beech forest, on dead branches of Fagus sylvatica, $41^{\circ} 20^{\prime} 43^{\prime \prime} \mathrm{N} 23^{\circ} 11^{\prime} 16^{\prime \prime} \mathrm{E}$, alt. 1310 m, 27 May 2010, leg. A. Atanassova 110202 \& H. Mayrhofer; same area, on Acer heldreichii,

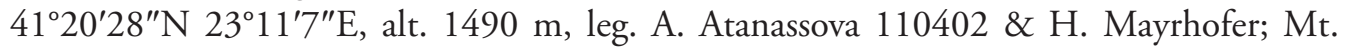
Slavyanka, Blagoevgrad Province, Izvora Hut, SW of Petrovo village, on Ailanthus altissima, $41^{\circ} 24^{\prime} 53^{\prime \prime N} 23^{\circ} 33^{\prime} 27^{\prime \prime} \mathrm{E}$, alt. 707 m, 28 May 2010, leg. A. Atanassova 120317 \& H. Mayrhofer; Goleshovo village, open pasture with scattered old deciduous trees, on Acer

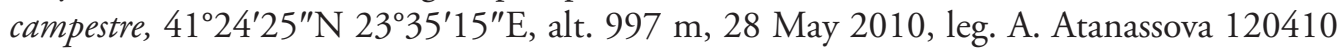
\& H. Mayrhofer.

*Rinodina teichophila (Nyl.) Arnold [17]

The Rhodopes, Plovdiv Province, SE of Novakovo village, deciduous forest edge, on exposed

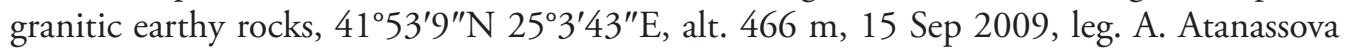
175406.

\section{${ }^{*}$ Rinodina trevisanii (Hepp) Körb. [15]}

Rila Mts, Kyustendil Province, Panichishte Resort, mixed forest and pasture along the road, on roots of Pinus sylvestris, $42^{\circ} 16^{\prime} 57^{\prime \prime} \mathrm{N} 23^{\circ} 16^{\prime} 15^{\prime} \mathrm{E}$, alt. $875 \mathrm{~m}, 24$ May 2010, leg. A. Atanassova $150301 \&$ H. Mayrhofer.

Rinodinella controversa (A. Massal.) H. Mayrhofer \& Poelt [5, 18]

Balkan Range, Mt. Ponor, Sofia Province, Dobravitsa village, Topilata neighbourhood, on limestone, parasitic on Rinodina lecanorina, $43^{\circ} 1^{\prime} 39^{\prime \prime} \mathrm{N} 23^{\circ} 14^{\prime} 39^{\prime \prime} \mathrm{E}$, alt. $1100 \mathrm{~m}, 15$ Jul 2007, leg. H. Mayrhofer 18625 \& S. Nikolova; Thracian Lowland, Plovdiv Province, Besaparski Ridove, on limestone, 42 $5^{\prime} 13^{\prime \prime} \mathrm{N} 24^{\circ} 28^{\prime} 47^{\prime \prime} \mathrm{E}$, alt. 220 m, 13 May 2008, leg. H. Mayrhofer 18566 \& S. Nikolova. 
${ }^{*}$ Rinodinella dubyanoides (Hepp) H. Mayrhofer \& Poelt [6]

Sofia Region, Sofia Province, Beledie Han, on limestone, 42 $53^{\prime} 27^{\prime \prime} \mathrm{N} 23^{\circ} 9^{\prime} 48^{\prime \prime} \mathrm{E}$, alt. 770 m, 15 Jul 2007, leg. H. Mayrhofer 18588 \& S. Nikolova.

Schaereria fuscocinerea (Nyl.) Clauzade \& Cl. Roux [11]

Mt. Belasitsa, Blagoevgrad Province, Kongour Peak, earthy outcrops, on silicate, $41^{\circ} 19^{\prime} 16^{\prime \prime} \mathrm{N}$ $23^{\circ} 10^{\prime 2} 9^{\prime \prime}$, alt. 1951 m, 27 May 2010, leg. A. Atanassova 110802 \& H. Mayrhofer.

Schismatomma pericleum (Ach.) Branth \& Rostr. [14]

Pirin Mts, Blagoevgrad Province, along the road to Bunderitsa Hut, on Abies alba, $41^{\circ} 46^{\prime} 35^{\prime \prime} \mathrm{N} 23^{\circ} 26^{\prime} 21^{\prime \prime} \mathrm{E}$, alt. $1751 \mathrm{~m}, 9$ Jul 2007, leg. H. Mayrhofer 18686 \& S. Nikolova.

Scoliciosporum chlorococcum (Graewe ex Stenh.) Vězda [5]

Balkan Range, Mt. Ponor, Sofia Province, Dobravitsa village, Topilata neighbourhood, on Juglans regia, 431'39"N 2314'39"E, alt. 1096 m, 15 Jul 2007, leg. H. Mayrhofer \& S. Nikolova (SOMF 26407).

* Scoliciosporum umbrinum var. corticicolum (Anzi) Bagl. \& Carestia [9, 11]

West Frontier Mts, Mt. Vlahina, Blagoevgrad Province, SW of Padesh village, near a small riverbed, on Populus sp., 41 ${ }^{\circ} 54^{\prime} 22^{\prime \prime} \mathrm{N} 22^{\circ} 57^{\prime} 50^{\prime \prime} \mathrm{E}$, alt. 655 m, 26 May 2010, leg. A. Atanassova 090317 \& H. Mayrhofer; Mt. Belasitsa, Blagoevgrad Province, near Belasitsa Hut, deciduous forest, on Betula pendula, 41 $22^{\prime} 9^{\prime \prime} \mathrm{N} 23^{\circ} 11^{\prime} 43^{\prime \prime} \mathrm{E}$, alt. 690 m, 27 May 2010, leg. A. Atanassova 110109 \& H. Mayrhofer.

\#Scytinium pulvinatum (Hoffm.) Otálora, P.M. Jørg. \& Wedin [17]

Published by Suza (1929: 10, as 'Leptogium lichenoides var. pulvinatum') and Szatala (1930: 68, as 'Leptogium pulvinatum'). Mayrhofer et al. (2005) treated these taxa as synonyms of Leptogium lichenoides.

\#Scytinium turgidum (Ach.) Otálora, P.M. Jørg. \& Wedin [5]

Published by Szatala (1929: 87, as 'Leptogium turgidum'). Mayrhofer et al. (2005) treated this taxon as synonym of Leptogium plicatile.

Solenopsora candicans (Dicks.) J. Steiner [17]

The Rhodopes, Smolyan Province, eco-path along Trigrad Gorge, on calcareous vertical

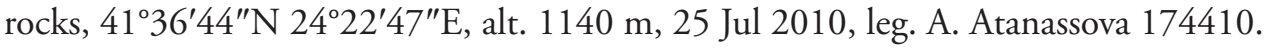

*Solorina bispora var. macrospora (Harm.) H. Olivier [14]

Pirin Mts, Blagoevgrad Province, near Mountain Shelter Kazana, on soil, $41^{\circ} 46^{\prime} 12^{\prime \prime} \mathrm{N}$ $23^{\circ} 24^{\prime 2} 24^{\prime \prime E}$, alt. 2408 m, 11 Jul 2007, leg. H. Mayrhofer 18649 \& S. Nikolova. 
*Strigula affinis (A. Massal.) R.C. Harris [17]

The Rhodopes, Haskovo Province, Kralevo village, in deciduous forest, on Quercus sp., $41^{\circ} 47^{\prime} 02^{\prime \prime} \mathrm{N} 25^{\circ} 40^{\prime} 55^{\prime \prime} \mathrm{E}$, alt. $330 \mathrm{~m}, 12$ Sep 2009, leg. A. Atanassova 175105.

Tephromela atra (Huds.) Hafellner var. atra [12]

Mt. Slavyanka, Blagoevgrad Province, SW of Petrovo village, Valley of Petrovska Reka River, S-facing open dry rocky slopes, on mica schist, $41^{\circ} 25^{\prime} 29^{\prime \prime} \mathrm{N} 23^{\circ} 31^{\prime} 27^{\prime \prime} \mathrm{E}$, alt. $534 \mathrm{~m}$, 28 May 2010, leg. A. Atanassova 120110 \& H. Mayrhofer.

* Tephromela atra var. torulosa (Flörke ex Flot.) Hafellner [14]

Pirin Mts, Blagoevgrad Province, between Vihren Hut and Bunderitsa Hut, on Pinus

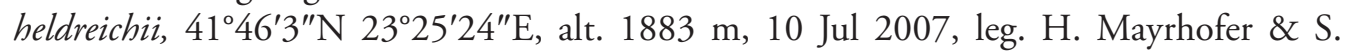
Nikolova (SOMF 26419); tourist road from Bunderitsa Hut to Kazanite Circus, on Pinus

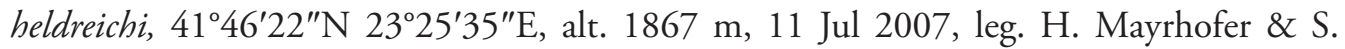
Nikolova (SOMF 26492).

Thalloidima opuntioides (Vill.) Kistenich et al. [17] (syn. Toninia opuntioides (Vill.) Timdal) The Rhodopes, Smolyan Province, Chudnite Mostove rock formation, on bryophytes, $41^{\circ} 49^{\prime} 06^{\prime \prime} \mathrm{N} 24^{\circ} 34^{\prime} 59^{\prime \prime} \mathrm{E}$, alt. 1403 m, 4 Sep 2010, leg. A. Atanassova 174004.

Thelenella modesta (Nyl.) Nyl. [10]

Valley of River Strouma, Blagoevgrad Province, W of Purvomay village, NW-facing open dry rocky slope with shrubs destructed by grazing stock, on Carpinus orientalis, $41^{\circ} 24^{\prime} 10^{\prime \prime} \mathrm{N}$ $23^{\circ} 6^{\prime} 48^{\prime \prime}$ E, alt. 185 m, 29 May 2010, leg. A. Atanassova 100730 \& H. Mayrhofer.

\section{Thrombium epigaeum (Pers.) Wallr. [11]}

Belasitsa Mt., Blagoevgrad Province, Kongour Peak, N-facing siliceous rock outcrops

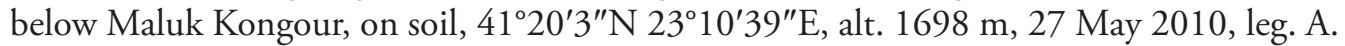
Atanassova 110609 \& H. Mayrhofer.

Toninia philippea (Mont.) Timdal [17]

The Rhodopes, Smolyan Province, near Trigrad, on breccia, $41^{\circ} 36^{\prime} 16^{\prime \prime} \mathrm{N} 24^{\circ} 22^{\prime} 40^{\prime \prime} \mathrm{E}$, alt. 1260 m, 16 May 2008, leg. H. Mayrhofer 18528 \& S. Nikolova; Kurdzhali Province, the dam of Stouden Kladenets Reservoir, on trachyte, 41 $36^{\prime} 59^{\prime \prime} \mathrm{N} 25^{\circ} 38^{\prime} 30^{\prime \prime E}$, alt. 230 m, 14 May 2008, leg. H. Mayrhofer 18540 \& S. Nikolova.

Trapeliopsis wallrothii (Flörke ex Spreng.) Hertel \& Gotth. Schneid. [15]

Rila Mts, Kyustendil Province, Panitschishte Resort, mixed forest and pasture along the road, on earth, $42^{\circ} 16^{\prime} 57^{\prime \prime} \mathrm{N} 23^{\circ} 16^{\prime} 15^{\prime \prime} \mathrm{E}$, alt. $875 \mathrm{~m}, 24$ May 2010, leg. A. Atanassova $150303 \&$ H. Mayrhofer. 
Umbilicaria crustulosa (Ach.) Frey [11]

Mt. Belasitsa, Blagoevgrad Province, Kongour Peak, on N-facing siliceous rock outcrops below Maluk Kongour, 41 ${ }^{\circ} 19^{\prime} 18^{\prime \prime} \mathrm{N} 23^{\circ} 10^{\prime} 28^{\prime \prime}$ E, alt. 1944 m, 27 May 2010, leg. A. Atanassova $110710 \& \mathrm{H}$. Mayrhofer.

*Umbilicaria freyi Codogno, Poelt \& Puntillo [14, 17]

Pirin Mts, Blagoevgrad Province, near Vihren Hut, on underhanging siliceous rock, $41^{\circ} 44^{\prime} 56^{\prime \prime} \mathrm{N} 23^{\circ} 24^{\prime} 48^{\prime \prime} \mathrm{E}$, alt. $2031 \mathrm{~m}, 10 \mathrm{Jul} 2007$, leg. H. Mayrhofer 18673 \& S. Nikolova, det. E.A. Davydov; the Rhodopes, Haskovo Province, near Madzharovo, steep N-facing rocks, on basalt, $41^{\circ} 38^{\prime} 24^{\prime \prime} \mathrm{N} 25^{\circ} 50^{\prime} 48^{\prime \prime} \mathrm{E}$, alt. $160 \mathrm{~m}, 14$ May 2008, leg. H. Mayrhofer 18178 \& S. Nikolova, det. E.A. Davydov.

Umbilicaria hirsuta (Sw. ex Westr.) Ach. [10]

Valley of River Strouma, Blagoevgrad Province, Kresna Gorge, on siliceous rocks, $41^{\circ} 45^{\prime} 48^{\prime \prime} \mathrm{N} 23^{\circ} 9^{\prime} 10^{\prime \prime} \mathrm{E}$, alt. $220 \mathrm{~m}, 12 \mathrm{Jul} 2007$, leg. H. Mayrhofer \& S. Nikolova (SOMF 26470).

*Umbilicaria maculata Krzew., M.P. Martín \& M.A. García [11, 15]

Mt. Belasitsa, Blagoevgrad Province, N-facing siliceous rock outcrops below Maluk Kongour, on mica schist, $41^{\circ} 19^{\prime} 18^{\prime \prime} \mathrm{N} 23^{\circ} 10^{\prime} 28^{\prime \prime} \mathrm{E}$, alt. $1944 \mathrm{~m}, 27$ May 2010, leg. A. Atanassova 110723 \& H. Mayrhofer, det. E. Davydov; Rila Mts, Kyustendil Province, Seven Rila Lakes, SW above the Dolnoto Ezero, on mica schist, 42 $12^{\prime} 58^{\prime \prime} \mathrm{N} 23^{\circ} 19^{\prime} 13^{\prime \prime} \mathrm{E}$, alt. $2158 \mathrm{~m}$, 25 May 2010, A. Atanassova 151003 \& H. Mayrhofer, det. E. Davydov.

Usnea substerilis Motyka [14]

Pirin Mts, Blagoevgrad Province, between Bunderitsa Hut and Vihren Hut, on Pinus peuce, $41^{\circ} 45^{\prime} 48^{\prime \prime} \mathrm{N} 23^{\circ} 25^{\prime} 13^{\prime \prime} \mathrm{E}$, alt. $1875 \mathrm{~m}, 10 \mathrm{Jul} 2007$, leg. H. Mayrhofer 18635 \& S. Nikolova.

Xanthoparmelia pulla (Ach.) O. Blanco et al. [10]

Valley of River Strouma, Blagoevgrad Province, W of Purvomay village, NW-facing open dry rocky slope, on mica schist, $41^{\circ} 24^{\prime} 10^{\prime \prime} \mathrm{N} 23^{\circ} 6^{\prime} 48^{\prime \prime} \mathrm{E}$, alt. $185 \mathrm{~m}, 29$ May 2010, leg. A. Atanassova $100717 \& \mathrm{H}$. Mayrhofer.

Xanthoparmelia stenophylla (Ach.) Ahti \& D. Hawksw. [9, 10]

West Frontier Mts, Mt. Vlahina, Blagoevgrad Province, Padesh village, SE-facing pasture

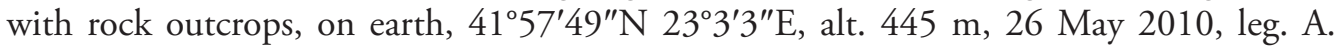
Atanassova 090106 \& H. Mayrhofer; Valley of River Strouma, Blagoevgrad Province, W of Purvomay village, NW-facing open dry rocky slope, on mica schist, $41^{\circ} 24^{\prime} 10^{\prime \prime} \mathrm{N} 23^{\circ} 6^{\prime} 48^{\prime \prime} \mathrm{E}$, alt. 185 m, 29 May 2010, leg. A. Atanassova 100712 \& H. Mayrhofer. 
Xanthoparmelia tinctina (Maheu \& A. Gillet) Hale [1]

Black Sea coast, Bourgas Province, Sinemorets village, estuary of Veleka River, on volcanic rocks, 42 $3^{\prime} 54^{\prime \prime} \mathrm{N} 27^{\circ} 58^{\prime} 33^{\prime \prime} \mathrm{E}$, alt. 10 m, 22 Sep 2010, leg. A. Atanassova 010807.

\#Xylographa pallens (Nyl.) Harm. [17]

Published by Szatala (1930: 65, as 'Xylographa abietina f. pallens'). Mayrhofer et al. (2005) treated this taxon as synonym of $X$. parallela.

\section{Lichenicolous fungus}

Carbonea vitellinaria ( $\mathrm{Nyl}$.) Hertel [11]

Mt. Belasitsa, Blagoevgrad Province, Kongour Peak, on N-facing siliceous rock outcrops

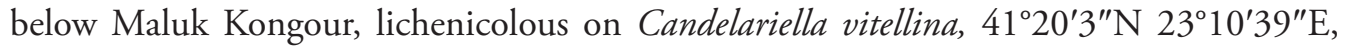
alt. 1698 m, 27 May 2010, leg. A. Atanassova 110604 \& H. Mayrhofer.

Host: Candelariella vitellina

Acknowledgements. We are grateful to Evgeny Davydov (Barnaul), Theodore Esslinger (Fargo), Josef Hafellner (Graz), Kerry Knudsen (Praha), Jiri Malíček (Praha), Fernando Fernandez Mendoza (Graz), Philipp Resl (Graz), and J. Vondrák (Praha) for the determination of selected specimens, and Peter Kosnik (Graz) for assistance with TLC. The study of C.M. Denchev was supported by the Bulgarian Science Fund (Grant no. KP-06 PN36/17/2019).

\section{References}

Gaya, E. 2009. Taxonomical revision of the Caloplaca saxicola group (Teloschistaceae, lichen-forming Ascomycota). - Bibliotheca Lichenologica 101: 1-191.

Hertel, H. 1971. Beiträge zur Kenntnis der Flechtenfamilie Lecideaceae IV. - Herzogia 2: 231-261.

Hertel, H. 2006. World distribution of species of Lecidea (Lecanorales) occurring in Central Europe. - In: A. Lackovičová, A. Guttová, E. Lisická \& P. Lizoň (eds). Central European lichens - diversity and threat, pp. 19-74. Mycotaxon Ltd, Ithaca, NY.

Kazandzhiev, S. 1900. [Lichen flora of Bulgaria]. - Periodichesko Spisanie na Bulgarskoto Knizhovno Drouzhestvo 61: 470-532. (In Bulgarian)

Mayrhofer, H., Denchev, C.M., Stoykov, D.Y. \& Nikolova, S.O. 2005. Catalogue of the lichenized and lichenicolous fungi in Bulgaria. - Mycologia Balcanica 2: 3-61.

http://doi.org/10.5281/zenodo.2546852

Nimis, P.L. 2016. The lichens of Italy. A second annotated catalogue. EUT, Trieste.

Nimis, P.L., Hafellner, J., Roux, C., Clerc, P., Mayrhofer, H., Martellos, S. \& Bilovitz, P.O. 2018. The lichen of the Alps - an annotated checklist. - MycoKeys 31: 1-634.

https://doi.org/10.3897/mycokeys.31.23568

Orange, A., James, P.W. \& White, F.J. 2001. Microchemical methods for the identification of lichens. British Lichen Society, London. 
Poelt, J. \& Vězda, A. 1981. Bestimmungsschlüssel europäischer Flechten. Ergänzungsheft II. - Bibliotheca Lichenologica 16: 1-390.

Popnikolov, A. \& Zhelezova, B. 1964. [Flora of Bulgaria. Lichens]. Narodna Prosveta, Sofia. (In Bulgarian) Šoun, J., Vondrák, J., Søchting, U., Hrouzek, P., Khodosovtsev, A. \& Arup, U. 2011. Taxonomy and phylogeny of the Caloplaca cerina group in Europe. - Lichenologist 43: 113-135.

https://doi.org/10.1017/S0024282910000721

Suza, J. 1929. Lichenes Bulgariae, I. - Acta Botanica Bohemica 8: 7-25.

Szatala, Ö. 1929. Beiträge zur Flechtenflora von Bulgarien. I. - Magyar Botanikai Lapok 28: 82-99.

Szatala, Ö. 1930. Beiträge zur Flechtenflora von Bulgarien. II. - Magyar Botanikai Lapok 29: 58-104.

Vondrák, J., Šoun, J., Redchenko, O., Lőkös, L. \& Khodosovtsev, A. 2009. Populations of two Caloplaca species with peculiar ecology observed in the Bükk Mts, Hungary. - Bryonora 44: 8-12. 